\title{
DARBOUX INTEGRABILITY AND ALGEBRAIC LIMIT CYCLES FOR A CLASS OF POLYNOMIAL DIFFERENTIAL SYSTEMS
}

\author{
JINLONG CAO ${ }^{1}$, JAUME LLIBRE ${ }^{2}$ AND XIANG ZHANG ${ }^{1}$
}

\begin{abstract}
This paper deals with the existence of Darboux first integrals for the planar polynomial differential systems $\dot{x}=\lambda x-y+P_{n+1}(x, y)+x F_{2 n}(x, y), \dot{y}=x+\lambda y+$ $Q_{n+1}(x, y)+y F_{2 n}(x, y)$, where $P_{i}(x, y), Q_{i}(x, y)$ and $F_{i}(x, y)$ are homogeneous polynomials of degree $i$. Inside this class we identify some new Darboux integrable systems having either a focus or a center at the origin. For such Darboux integrable systems having degrees 5 and 9 we give the explicit expressions of their algebraic limit cycles. For the systems having degrees $3,5,7$ and 9 we present necessary and sufficient conditions for being Darboux integrable.
\end{abstract}

\section{Introduction AND STATEMENT OF THE MAIN RESUlts}

There exist three main open problems in the qualitative theory of real planar differential systems, the distinction between a center and a focus, the determination of the number of limit cycles and their distribution, and the determination of its integrability. The importance for searching first integrals of a given system was already noted by Poincaré [24] in his discussion on a method to obtain polynomial or rational first integrals. One of the classical tools in the classification of all trajectories of a dynamical system is to find first integrals. In $[13,14]$ the authors characterized a large classes of polynomial differential systems in terms of the existence of Darboux first integrals. In this paper we will extend the results of $[13,14]$ to a new class of polynomial differential systems.

We study the following systems

$$
\begin{aligned}
& \frac{d x}{d t}=\dot{x}=\lambda x-y+P_{n+1}(x, y)+x F_{2 n}(x, y):=P(x, y), \\
& \frac{d y}{d t}=\dot{y}=x+\lambda y+Q_{n+1}(x, y)+y F_{2 n}(x, y):=Q(x, y),
\end{aligned}
$$

where $P_{i}(x, y), Q_{i}(x, y)$ and $F_{i}(x, y)$ are homogeneous polynomials of degree $i$ in the variables $x$ and $y$. These systems have a focus at the origin if $\lambda \neq 0$, and have either a center or a focus if $\lambda=0$. Recall that a center is a singular point having a neighborhood filled of periodic orbits, and that a focus is a singular point having a neighborhood where all the orbits spiral either in forward or in backward time to it. This work will characterize some new class of Darboux integrable systems.

2000 Mathematics Subject Classification. 34A34, 34C07.

Key words and phrases. Darboux first integral; algebraic limit cycles; Abel differential equation. 
Taking polar coordinates $(r, \theta)$ via $x=r \cos \theta$ and $y=r \sin \theta$, system (1) becomes

$$
\dot{r}=\lambda r+f(\theta) r^{n+1}+h(\theta) r^{2 n+1}, \quad \dot{\theta}=1+g(\theta) r^{n},
$$

where

$$
\begin{aligned}
f(\theta) & =\cos \theta P_{n+1}(\cos \theta, \sin \theta)+\sin \theta Q_{n+1}(\cos \theta, \sin \theta), \\
g(\theta) & =\cos \theta Q_{n+1}(\cos \theta, \sin \theta)-\sin \theta P_{n+1}(\cos \theta, \sin \theta), \\
h(\theta) & =F_{2 n}(\cos \theta, \sin \theta) .
\end{aligned}
$$

We remark that $f(\theta), g(\theta)$ and $h(\theta)$ are homogeneous trigonometric polynomials of degree $n+2, n+2$ and $2 n$, respectively. In the region $W=\left\{(r, \theta): 1+g(\theta) r^{n}>0\right\}$, system $(2)$ can be written as the differential equation

$$
\frac{d r}{d \theta}=\frac{\lambda r+f(\theta) r^{n+1}+h(\theta) r^{2 n+1}}{1+g(\theta) r^{n}} .
$$

It can be transformed to the Abel differential equation

$$
\begin{aligned}
\frac{d \rho}{d \theta} & =n\left(h(\theta)-f(\theta) g(\theta)+\lambda g^{2}(\theta)\right) \rho^{3}+\left(n f(\theta)-g^{\prime}(\theta)-2 n \lambda g(\theta)\right) \rho^{2}+n \lambda \rho \\
& =A(\theta) \rho^{3}+B(\theta) \rho^{2}+C \rho
\end{aligned}
$$

via the diffeomorphism

$$
\rho=r^{n} /\left(1+g(\theta) r^{n}\right), \quad(r, \theta) \in W .
$$

As far as we know Cherkas [3] is the first one to use this transformation. The differential equation (4) appeared in the Abel's studies on the theory of elliptic functions (for more details on Abel differential equations, see for instance $[2,8,15,10])$.

In system (1), if we take $P_{n+1}(x, y)=x F_{n}(x, y)$ and $Q_{n+1}(x, y)=y F_{n}(x, y)$, system (2) has $g(\theta)=0$. These planar systems whose angular speed is constant are usually called rigid or uniformly isochronous (see e.g. [11]). Of course, for these differential systems all their centers are isochronous, see e.g. [7, 26]. Giné and Llibre [14] have studied the system with $g(\theta)=0$ and characterized its Darboux integrability. If we take $F_{2 n}(x, y)=0$ in system (1), the system has homogeneous nonlinearities. The Darboux integrability of these systems was studied in [13], and in $[17,18]$ the existence of limit cycles was analyzed.

Our system can have $g(\theta)$ and $h(\theta)$ both non-vanishing. Also as those in [13, 14] our system (1) can have Darboux first integrals and algebraic limit cycles.

We shall consider the Abel differential equation (4) defined on the cylinder $(\rho, \theta) \in$ $\mathbb{R} \times \mathbb{S}^{1}$, where $\mathbb{R}$ is the field of real numbers, and $\mathbb{S}^{1}$ is the unit circle. Of course only the orbits of the half-cylinder $\rho>0$ can come from the ones of system (1). Note that the origin of system (1) plays the role of the periodic orbit $\rho=0$ for the Abel differential equation (4).

We say that all polynomial differential systems of form (1) define a subclass $\mathcal{J}$ if and only if $A(\theta)$ and $B(\theta)$ satisfy

$$
A^{\prime}(\theta) B(\theta)-A(\theta) B^{\prime}(\theta)=a B^{3}(\theta)-A(\theta) B(\theta) C
$$


for some $a \in \mathbb{R}$ and $C=n \lambda$. In this paper we shall prove that each polynomial differential system (1) in the subclass $\mathcal{J}$ has a Darboux first integral, and show by examples that some of them can have one or two algebraic limit cycles.

Denote by

$$
\mathcal{X}=P(x, y) \frac{\partial}{\partial x}+Q(x, y) \frac{\partial}{\partial y},
$$

the vector field associated to system (1). Let $\Sigma$ be the set of some special orbits of system (1) such that $U \subset \mathbb{R}^{2} \backslash \Sigma$ is open. System (1) is smooth (or analytic) integrable on $U$ if there exists a nonconstant smooth (or analytic) function $H: U \rightarrow \mathbb{R}$, called a first integral of the system on $U$, which is constant on all solution curves $(x(t), y(t))$ of system (1) on $U$; i.e. $H(x(t), y(t))=$ constant for all values of $t$ for which the solution is defined on $U$. Clearly $H$ is a smooth first integral of system (1) on $U$ if and only if $\mathcal{X} H \equiv 0$ on $U$.

We say that a $C^{k}$ function $R: U \rightarrow \mathbb{R}$ with $k \geq 1$, not identically null in $U$, is an integrating factor of system (1) in $U$ if $\partial(R P) / \partial x=-\partial(R Q) / \partial y$. In this case the first integral $H$ associated to this integrating factor $R$ is given by

$$
H(x, y)=-\int R(x, y) P(x, y) \mathrm{d} y+f(x),
$$

satisfying $\partial H / \partial x=R Q$. Then $\dot{x}=R P=-\partial H / \partial y$ and $\dot{y}=R Q=\partial H / \partial x$.

The following result, due to Giacomini et al [12], is very useful in studying the existence or nonexistence of limit cycles.

Theorem 1. Let $(P, Q)$ be a $C^{1}$ vector field defined in the open subset $U$ of $\mathbb{R}^{2}$. Let $V(x, y)$ be a solution of the linear partial differential equation

$$
P \frac{\partial V}{\partial x}+Q \frac{\partial V}{\partial y}=\left(\frac{\partial P}{\partial x}+\frac{\partial Q}{\partial y}\right) V
$$

defined in $U$. If $\gamma$ is a limit cycle of $(P, Q)$ contained in $U$, then $\gamma$ is contained in $\{(x, y) \in$ $U: V(x, y)=0\}$.

A nonconstant $C^{k}$ function $V: U \rightarrow \mathbb{R}$ with $k \geq 1$ satisfying (7) is called an inverse integrating factor of the vector field $\mathcal{X}$ in $U$. The expression $\operatorname{div}(\mathcal{X})=\operatorname{div}(P, Q)=\partial P / \partial x+$ $\partial Q / \partial y$ is called divergence of system (1). The function $R=1 / V$ defines in $U \backslash\{V=0\}$ an integrating factor of system (1) which allows to determine a first integral for system (1) in $U \backslash\{V=0\}$ (see e.g. $[5,1]$ ).

A function of the form $f_{1}^{\lambda_{1}} \ldots f_{p}^{\lambda_{p}}\left(\exp \left(h_{1} / g_{1}\right)\right)^{\mu_{1}} \ldots\left(\exp \left(h_{m} / g_{m}\right)\right)^{\mu_{m}}$, where the polynomials $f_{i}$ are irreducible in $\mathbb{C}[x, y]$ for $i=1, \ldots, p$, and the polynomials $g_{j}$ and $h_{j}$ are coprime in $\mathbb{C}[x, y]$ for $j=1, \ldots, m$, and the $\lambda_{i}$ and $\mu_{j}$ are complex numbers, is called a Darboux function. System (1) is called Darboux integrable if the system has a first integral or an integrating factor which is a Darboux function (see e.g. [20, 29]).

Our main results are the following

Theorem 2. For system (1) belonging to $\mathcal{J}$ the following statements hold. 
(a) If $\lambda \neq 0, a \neq 0$ and $A(\theta) B(\theta) \neq 0$, then the origin is a focus and the system has the Darboux first integral $\bar{H}(x, y)$ given by

$$
H(\rho, \theta)= \begin{cases}\frac{\rho \exp (-n \lambda \theta) \exp \left(-\frac{1}{\sqrt{4 a-1}} \arctan \frac{B(\theta)+2 \rho A(\theta)}{\sqrt{4 a-1} B(\theta)}\right)}{\sqrt{\rho^{2} A^{2}(\theta) / B^{2}(\theta)+\rho A(\theta) / B(\theta)+a}} & \text { if } a>\frac{1}{4}, \\ \frac{\rho B(\theta) \exp (-n \lambda \theta) \exp \frac{B(\theta)}{B(\theta)+2 \rho A(\theta)}}{B(\theta)+2 \rho A(\theta)} & \text { if } a=\frac{1}{4}, \\ \frac{\rho \exp (-n \lambda \theta)|\sqrt{1-4 a}+1+2 \rho A(\theta) / B(\theta)|^{(-1+1 / \sqrt{1-4 a}) / 2}}{|\sqrt{1-4 a}-1-2 \rho A(\theta) / B(\theta)|^{(1+1 / \sqrt{1-4 a}) / 2}} & \text { if } a<\frac{1}{4},\end{cases}
$$

through the changes of variables $x=r \cos \theta, y=r \sin \theta$ and the transformation (5).

(b) If $\lambda \neq 0$ and $A(\theta)=0$ or $B(\theta)=0$, but $A(\theta)$ and $B(\theta)$ do not vanish simultaneously, then the origin is a focus and the system has the Darboux first integral $\bar{H}(x, y)$ obtained from

$$
H(\rho, \theta)= \begin{cases}\exp (n \lambda \theta) \rho^{-1}+\int \exp (n \lambda \theta) B(\theta) \mathrm{d} \theta & \text { if } A(\theta)=0, \\ \exp (2 n \lambda \theta) \rho^{-2}+2 \int \exp (2 n \lambda \theta) A(\theta) \mathrm{d} \theta & \text { if } B(\theta)=0,\end{cases}
$$

through the changes of variables $x=r \cos \theta, y=r \sin \theta$ and the transformation (5).

(c) If $\lambda \neq 0$ and $A(\theta)=B(\theta)=0$, then the origin is a focus and the system has the Darboux first integral $\bar{H}(x, y)$ obtained from $H(\rho, \theta)=\rho \exp (-n \lambda \theta)$.

(d) If $\lambda=0$, then the origin is a center. Moreover the system has an explicit rational first integral if one of the following conditions holds.

$\left(d_{1}\right) A(\theta)=0$ and $B(\theta)=0$;

$\left(d_{2}\right) A(\theta)=0, B(\theta) \neq 0$ and $\int_{0}^{2 \pi} B(\theta) d \theta=0$;

$\left(d_{3}\right) B(\theta)=0, A(\theta) \neq 0$ and $\int_{0}^{2 \pi} A(\theta) d \theta=0$;

(d $\left.d_{4}\right) A(\theta) B(\theta) \neq 0$, and the parameter a given in (6) satisfies $a<1 / 4, a \neq 0$, and $\sqrt{1-4 a}$ rational.

We remark that the case $\lambda \neq 0, a \neq 0$ and $A(\theta) B(\theta)=0$ is contained in statement $(b)$, which can be seen from equation (6). Also if $\lambda \neq 0$ and $a=0$, we can prove that system (2) belongs to the subclass $\mathcal{J}$ if and only if $A(\theta)=0$ or $B(\theta)=0$. This case belongs to statement $(b)$, too. Theorem 2 will be proved in Section 2 .

A limit cycle of system (1) is a periodic orbit isolated in the set of all periodic orbits of system (1). We say that a limit cycle is algebraic if it is contained in an algebraic curve (see for instance, $[27,13])$.

Using Theorems 1 and 2, the following corollary follows easily from the fact that

$$
V(\rho, \theta)=\rho\left(\rho^{2} A^{2}(\theta) / B^{2}(\theta)+\rho A(\theta) / B(\theta)+a\right)
$$


is an inverse integrating factor of the Abel differential equation (4) in the subclass $\mathcal{J}$ with $\lambda \neq 0$ and $A(\theta) B(\theta) \neq 0$.

Corollary 3. If $n$ is even and $a \leq 1 / 4$ system (1) in the subclass $\mathcal{J}$ with $\lambda \neq 0$ and $A(\theta) B(\theta) \neq 0$ can have at most two limit cycles in the region $\dot{\theta}>0$, which are algebraic (if exist). Moreover there exist examples showing that some of these systems can have 1 or 2 limit cycles.

We now apply our theory to characterize Darboux integrable systems of form (1) with $n=1,2,3,4$.

System (1) with $n=1$ is of the form

$$
\begin{aligned}
& \dot{x}=-y+\lambda_{0} x+\lambda_{1} x^{2}+\lambda_{2} x y+\lambda_{3} y^{2}+x\left(\lambda_{7} x^{2}+\lambda_{8} x y+\lambda_{9} y^{2}\right), \\
& \dot{y}=x+\lambda_{0} y+\lambda_{4} x^{2}+\lambda_{5} x y+\lambda_{6} y^{2}+y\left(\lambda_{7} x^{2}+\lambda_{8} x y+\lambda_{9} y^{2}\right),
\end{aligned}
$$

which is a projective quadratic system (see e.g. $[19,22,28]$ ) because extended to the real projective plane $\mathbb{R} P^{2}$ continues being quadratic, where $\lambda_{i}, i=0, \cdots, 9$, are arbitrary constants. System (8) for $\lambda_{3}=\lambda_{4}=0, \lambda_{1}=\lambda_{5}$ and $\lambda_{2}=\lambda_{6}$ was studied by Collins in [6]. The Darboux integrability of system (8) was studied in [14] under the condition $\lambda_{3}=\lambda_{4}=0, \lambda_{1}=\lambda_{5}$ and $\lambda_{2}=\lambda_{6}$, and in [13] under the condition $\lambda_{7}=\lambda_{8}=\lambda_{9}=0$. In Corollary 4 of Section 3 we provide new classes of Darboux integrable systems of form (8). In Corollary 9 of Appendix $A$ we provide the necessary and sufficient conditions in order that system (8) with $\lambda_{3}=\lambda_{4}=0$ be in the subclass $\mathcal{J}$.

System (1) with $n=2$ is of the form

$$
\begin{aligned}
\dot{x}=-y+ & \lambda_{0} x+\lambda_{1} x^{3}+\lambda_{2} x^{2} y+\lambda_{3} x y^{2}+\lambda_{4} y^{3} \\
& +x\left(\lambda_{9} x^{4}+\lambda_{10} x^{3} y+\lambda_{11} x^{2} y^{2}+\lambda_{12} x y^{3}+\lambda_{13} y^{4}\right), \\
\dot{y}=x+ & \lambda_{0} y+\lambda_{5} x^{3}+\lambda_{6} x^{2} y+\lambda_{7} x y^{2}+\lambda_{8} y^{3} \\
& +y\left(\lambda_{9} x^{4}+\lambda_{10} x^{3} y+\lambda_{11} x^{2} y^{2}+\lambda_{12} x y^{3}+\lambda_{13} y^{4}\right),
\end{aligned}
$$

where $\lambda_{i}, i=0, \cdots, 13$, are arbitrary constants. For $\lambda_{4}=\lambda_{5}=0, \lambda_{1}=\lambda_{6}, \lambda_{2}=\lambda_{7}$ and $\lambda_{3}=\lambda_{8}$, the system has a Darboux first integral [14], and its origin is a center [26]. Systems (9) with $\lambda_{9}=\cdots=\lambda_{13}=0$ have also Darboux first integrals [13]. In Corollary 5 of Section 3 we provide new subclasses of Darboux integrable systems of form (9). In Proposition 6 of Section 4 we present the explicit expressions of the algebraic limit cycles contained in the region $\dot{\theta}>0$ of these systems with $\lambda_{4}=\lambda_{5}=0$. In Corollary 10 of Appendix $A$ we provide the necessary and sufficient conditions in order that system (9) with $\lambda_{4}=\lambda_{5}=0$ be in the subclass $\mathcal{J}$. 
System (1) with $n=3$ can be written in

$$
\begin{aligned}
\dot{x}= & -y+\lambda_{0} x+\lambda_{1} x^{4}+\lambda_{2} x^{3} y+\lambda_{3} x^{2} y^{2}+\lambda_{4} x y^{3}+\lambda_{5} y^{4} \\
& +x\left(\lambda_{11} x^{6}+\lambda_{12} x^{5} y+\lambda_{13} x^{4} y^{2}+\lambda_{14} x^{3} y^{3}+\lambda_{15} x^{2} y^{4}+\lambda_{16} x y^{5}+\lambda_{17} y^{6}\right), \\
\dot{y}= & x+\lambda_{0} y+\lambda_{6} x^{4}+\lambda_{7} x^{3} y+\lambda_{8} x^{2} y^{2}+\lambda_{9} x y^{3}+\lambda_{10} y^{4} \\
& +y\left(\lambda_{11} x^{6}+\lambda_{12} x^{5} y+\lambda_{13} x^{4} y^{2}+\lambda_{14} x^{3} y^{3}+\lambda_{15} x^{2} y^{4}+\lambda_{16} x y^{5}+\lambda_{17} y^{6}\right),
\end{aligned}
$$

where $\lambda_{i}, i=0, \cdots, 17$, are arbitrary constants. In Corollary 11 of Appendix $A$ we provide the necessary and sufficient conditions in order that system (10) with $\lambda_{5}=\lambda_{6}=0$ be in the subclass $\mathcal{J}$.

System (1) with $n=4$ can be written as

$$
\begin{aligned}
\dot{x}= & -y+\lambda_{0} x+\lambda_{1} x^{5}+\lambda_{2} x^{4} y+\lambda_{3} x^{3} y^{2}+\lambda_{4} x^{2} y^{3}+\lambda_{5} x y^{4}+\lambda_{6} y^{5}+x\left(\lambda_{13} x^{8}\right. \\
& \left.+\lambda_{14} x^{7} y+\lambda_{15} x^{6} y^{2}+\lambda_{16} x^{5} y^{3}+\lambda_{17} x^{4} y^{4}+\lambda_{18} x^{3} y^{5}+\lambda_{19} x^{2} y^{6}+\lambda_{20} x y^{7}+\lambda_{21} y^{8}\right), \\
\dot{y}= & x+\lambda_{0} y+\lambda_{7} x^{5}+\lambda_{8} x^{4} y+\lambda_{9} x^{3} y^{2}+\lambda_{10} x^{2} y^{3}+\lambda_{11} x y^{4}+\lambda_{12} y^{5}+y\left(\lambda_{13} x^{8}\right. \\
& \left.+\lambda_{14} x^{7} y+\lambda_{15} x^{6} y^{2}+\lambda_{16} x^{5} y^{3}+\lambda_{17} x^{4} y^{4}+\lambda_{18} x^{3} y^{5}+\lambda_{19} x^{2} y^{6}+\lambda_{20} x y^{7}+\lambda_{21} y^{8}\right),
\end{aligned}
$$

where $\lambda_{i}, i=0, \cdots, 21$, are arbitrary constants. In Proposition 8 of Section 4 we present the explicit expressions of the algebraic limit cycles contained in the region $\dot{\theta}>0$ of system (11) with $\lambda_{6}=\lambda_{7}=0$. In Corollary 12 of Appendix $A$ we obtain the necessary and sufficient conditions in order that system (11) with $\lambda_{6}=\lambda_{7}=0$ be in the subclass $\mathcal{J}$.

The rest of this paper is organized as follows. Section 2 is dedicated to prove Theorem 2. In Section 3 we present some new classes of Darboux integrable systems of form (8) and (9) belonging to the subclass $\mathcal{J}$. We give the explicit expressions of the algebraic limit cycles contained in the region $\dot{\theta}>0$ for quintic and nine degree systems having a Darboux first integral in Section 4. Finally, in Appendix A we present the necessary and sufficient conditions for systems (8), (9), (10) and (11) having $g(\theta)=0$ to be in the subclass $\mathcal{J}$.

\section{Proof of Theorem 2}

In this section we prove Theorem 2. The main idea follows from $[13,14]$.

(a) Following the case $(d)$ of Abel differential equation studied in [15], we take the change of variables $(\rho, \theta) \rightarrow(\eta, \xi)$ defined by $\rho=u(\theta) \eta(\xi)$, where $u(\theta)=\exp \left(\int n \lambda \mathrm{d} \theta\right)$ and $\xi=$ $\int u(\theta) B(\theta) \mathrm{d} \theta$. By this transformation the Abel differential equation (4) can be written as

$$
\eta^{\prime}(\xi)=g(\xi)[\eta(\xi)]^{3}+[\eta(\xi)]^{2},
$$

where $g(\xi)=u(\theta) A(\theta) / B(\theta)$ and ${ }^{\prime}=d / d \xi$.

Taking the change $\xi \rightarrow t$ as a new independent variable via $\xi^{\prime}=-1 /(t \eta(\xi))$, where $'=d / d t$ is the derivative with respect to $t$, equation (12) becomes

$$
t^{2} \xi^{\prime \prime}(t)+g(\xi(t))=0 .
$$


This nonlinear ordinary differential equation is a celebrated Euler differential equation if we take the particular case that $g(\xi)=a \xi$, where $a$ is an arbitrary constant. Then, equation (13) has the characteristic equation $k^{2}-k+a=0$. Therefore its general solution is

$$
\xi(t)= \begin{cases}C_{1} \sqrt{t}+C_{2} \sqrt{t} \ln t & \text { if } a=1 / 4, \\ C_{1} t^{k_{1}}+C_{2} t^{k_{2}} & \text { if } a \neq 1 / 4,\end{cases}
$$

where $k_{1}$ and $k_{2}$ are the roots of the characteristic equation.

We note that $g(\xi)=a \xi$ with $a \neq 0$ means

$$
\exp (n \lambda \theta) A(\theta) / B(\theta)=a \int \exp (n \lambda \theta) B(\theta) \mathrm{d} \theta
$$

or equivalently derivating (14) with respect to $\theta$ we get

$$
\frac{d}{d \theta} \frac{A(\theta)}{B(\theta)}=a B(\theta)-\frac{n \lambda A(\theta)}{B(\theta)},
$$

which is equivalent to condition (6). Finally returning to the variables $(\rho, \theta)$ and taking into account that the roots $k_{1}$ and $k_{2}$ can be real or complex, we obtain the first integrals of statement $(a)$ according to the values of $a$.

Now we shall prove that the systems of statement $(a)$ are Darboux integrable. For doing that it is sufficient to show that all the terms that appear in the first integral of those systems are of the form $f_{1}^{\lambda_{1}} \ldots f_{p}^{\lambda_{p}}$ where the $f_{i}$ 's are polynomials in $\mathbb{C}[x, y]$ and the $\lambda_{i}$ 's are complex numbers. First we have that

$$
\exp (-n \lambda \theta)=(\exp (2 \mathrm{i} \arctan (\sin \theta / \cos \theta)))^{n \lambda \mathrm{i} / 2}=(\cos \theta+\mathrm{i} \sin \theta)^{n \lambda \mathrm{i} / 2}(\cos \theta-\mathrm{i} \sin \theta)^{-n \lambda \mathrm{i} / 2},
$$

where we have used the fact $(\cos \theta+i \sin \theta)(\cos \theta-i \sin \theta)^{-1}=(\cos \theta+i \sin \theta)^{2}$. If among the exponential factors of systems (2) a complex conjugate pair $\exp (h / g)$ and $\exp (\bar{h} / \bar{g})$ occurs, the first integral has a real factor of the form

$$
(\exp (h / g))^{\mu}(\exp (\bar{h} / \bar{g}))^{\bar{\mu}}=\exp (2 \operatorname{Re}(\mu h / g)) .
$$

We recall that for $f \in \mathbb{C}[x, y]$, if $f=0$ is an invariant algebraic curve of a real polynomial differential system, then its complex conjugate $\bar{f}=0$ is also an invariant algebraic curve (see e.g. $[5,21]$ ). Therefore if among the invariant algebraic curves of systems (1) a complex conjugate pair $f=0$ and $\bar{f}=0$ occurs, then the first integral has a factor of the form $f^{\mu} \bar{f}^{\bar{\mu}}$, which is the (multi-valued) real function

$$
f^{\mu} \bar{f}^{\bar{\mu}}=\left[(\operatorname{Re} f)^{2}+(\operatorname{Im} f)^{2}\right]^{\operatorname{Re} \mu} \exp \left(-2 \operatorname{Im} \mu \arctan \left(\frac{\operatorname{Im} f}{\operatorname{Re} f}\right)\right) .
$$

On the other hand using $\rho=r^{n} /\left[1+g(\theta) r^{n}\right]$ it follows that

$$
F:=1+\frac{2 \rho A(\theta)}{B(\theta)}=\frac{r^{n+4}\left(B(\theta)+B(\theta) g(\theta) r^{n}+2 r^{n} A(\theta)\right)}{r^{n+4} B(\theta)\left(1+g(\theta) r^{n}\right)}
$$

is a rational function in cartesian coordinates because $A(\theta)$ consists of the homogeneous trigonometric polynomials of degree $2 n$ and $2 n+4$ in $\cos \theta$ and $\sin \theta$, and $B(\theta)$ and $g(\theta)$ are 
homogeneous of degree $n+2$ in $\cos \theta$ and $\sin \theta$. Hence, taking into account these relations the first integral for $a>1 / 4$ is given by the Darboux function

$$
H(\rho, \theta)=\rho \exp (-n \lambda \theta) f^{\mu} \bar{f}^{\bar{\mu}}
$$

where $\operatorname{Re} f=F, \operatorname{Im} f=\sqrt{4 a-1}, \operatorname{Re} \mu=-1 / 2$ and $\operatorname{Im} \mu=1 /(2 \sqrt{4 a-1})$. The first integral for $a=1 / 4$ is the Darboux function

$$
H(\rho, \theta)=\rho \exp (-n \lambda \theta) \exp (1 / F) / F .
$$

The first integral for $a<1 / 4$ and $a \neq 0$ is the Darboux function

$$
H(\rho, \theta)=\rho \exp (-n \lambda \theta)|\sqrt{1-4 a}+F|^{\mu_{1}}|\sqrt{1-4 a}-F|^{\mu_{2}} .
$$

where $\mu_{1}=(-1+1 / \sqrt{1-4 a}) / 2$ and $\mu_{2}=-(1+1 / \sqrt{1-4 a}) / 2$. This completes the proof of statement $(a)$.

(b) If $A(\theta)=0$ and $B(\theta) \neq 0$, or $A(\theta) \neq 0$ and $B(\theta)=0$, the Abel differential equation

(4) is the Bernoulli one $d \rho / d \theta=B(\theta) \rho^{2}+n \lambda \rho$, or $d \rho / d \theta=A(\theta) \rho^{3}+n \lambda \rho$. Solving these Bernoulli equations we get the first integrals of statement $(b)$. Systems (1) of statement $(b)$ are Darboux integrable because their first integrals are obtained by integrating elementary functions (for more details see [25]). Consequently these systems have a Darboux first integral taking into account that the integrals which appear in statement (b) can be computed using recurrent formulas (see for instance [23]).

(c) If $\lambda \neq 0$ and $A(\theta)=B(\theta)=0$, the Abel differential equation (4) is the linear differential equation $d \rho / d \theta=n \lambda \rho$. Solving this linear equation we get the first integrals of statement $(c)$. Systems (1) of statement $(c)$ are also Darboux integrable because their first integrals are obtained by integrating elementary functions.

(d) If $\lambda=0$ systems (1) belonging to the subclass $\mathcal{J}$ have the first integrals $\bar{H}(x, y)$ obtained from the expressions of $H(\rho, \theta)$ in statements $(a)$ and $(b)$ with $\lambda=0$. The first integrals $H(\rho, \theta)$ with $\lambda=0$ of $(a)$ and $(b)$ are all periodic of period $2 \pi$. So the origin is a center. Moreover from the Poincaré theorem [24] and the fact that the origin is a linear center, the system has a local analytic first integrals in a neighborhood of the origin. Usually we cannot compute this local analytic first integral.

If $B(\theta)=A(\theta)=0$, then system (4) has the first integral $\rho$. Consequently system (2) with $\lambda=0$ has the first integral $H_{1}=r^{2 n} /\left(1+g(\theta) r^{n}\right)^{2}$. Going back to the cartesian coordinates, it is a rational first integral of (1) because $g(\theta)$ is a homogeneous trigonometric polynomial of degree $n+2$ in $\cos \theta$ and $\sin \theta$. Statement $\left(d_{1}\right)$ follows.

If $A(\theta)=0$ and $B(\theta) \neq 0$, then system (4) has the first integral $H_{2}(\rho, \theta)=1 / \rho+$ $\int B(\theta) \mathrm{d} \theta$. The assumption $\int_{0}^{2 \pi} B(\theta) \mathrm{d} \theta=0$ implies that $\left(H_{2}(\rho, \theta)\right)^{2}$ is a rational function in cartesian coordinates. We note that if $n$ is even, so is $H_{2}(\rho, \theta)$. Hence system (1) has a rational first integral and consequently statement $\left(d_{2}\right)$ follows if $A(\theta)=0$ and $B(\theta) \neq 0$.

If $B(\theta)=0$ and $A(\theta) \neq 0$, then system (4) has the first integral $H_{3}(\rho, \theta)=1 / \rho^{2}+$ $2 \int A(\theta) \mathrm{d} \theta$. The assumption $\int_{0}^{2 \pi} A(\theta) \mathrm{d} \theta=0$ implies that $H_{3}(\rho, \theta)$ is a rational function 
in cartesian coordinates because $\rho^{2}$ is rational in the cartesian coordinates and $A(\theta)$ is a trigonometric polynomial of even degree in $\cos \theta$ and $\sin \theta$. Hence system (1) has a rational first integral and consequently statement $\left(d_{3}\right)$ follows if $B(\theta)=0$ and $A(\theta) \neq 0$.

Finally from the expression of the first integral $H(\rho, \theta)$ of statement $(a)$ for $a<1 / 4$, $a \neq 0$ and $\sqrt{1-4 a}$ rational, we have $H^{2}(\rho, \theta)=\rho^{2}|\sqrt{1-4 a}+F|^{2 \mu_{1}}|\sqrt{1-4 a}-F|^{2 \mu_{2}}$, where $\mu_{1}, \mu_{2}$ and $F$ are defined at the end of the proof of statement $(a)$. Then statement $\left(d_{4}\right)$ follows from the fact that $F$ is rational in the cartesian coordinates. This completes the proof of statement $(d)$.

\section{Darboux integrability of systems (8) And (9)}

The following corollary characterizes partially the Darboux integrability of system (8) belonging to the subclass $\mathcal{J}$.

Corollary 4. System (8) with $\lambda_{0} \neq 0$ belongs to the subclass $\mathcal{J}$ if one of the following statements holds (we note that $\lambda_{7}, \lambda_{8}$ and $\lambda_{9}$ can be obtained from condition (6).

(a) If $\lambda_{2}=\lambda_{4}=0, \lambda_{3}=-\lambda_{1}, \lambda_{6}=\lambda_{1}, \lambda_{5}=2 \lambda_{1}, a=\left(1+\lambda_{0}^{2}\right) /\left(4 \lambda_{0}^{2}\right)$, then system (8) has the Darboux first integral

$$
H(x, y)=\frac{\rho(x, y) \exp \left(-\lambda_{0} \arctan (y / x)-\lambda_{0} \arctan \lambda_{0}(1+2 \Gamma(x, y))\right)}{\sqrt{\Gamma^{2}(x, y)+\Gamma(x, y)+\left(1+\lambda_{0}^{2}\right) /\left(4 \lambda_{0}^{2}\right)}},
$$

with

$\Gamma(x, y)=\frac{-\lambda_{1}}{\lambda_{0}^{3} g_{0}}\left(\gamma_{6}^{a} x^{6}+\gamma_{5}^{a} x^{5} y+\gamma_{4}^{a} x^{4} y^{2}+\gamma_{3}^{a} x^{3} y^{3}+\gamma_{2}^{a} x^{2} y^{4}+\gamma_{1}^{a} x y^{5}+\gamma_{0}^{a} y^{6}\right)$,

$\rho(x, y)=-\left(x^{2}+y^{2}\right)^{3 / 2} /\left(\left(x^{2}+y^{2}\right)\left(1+\lambda_{1} y\right)+\lambda_{1} x y^{2}\right)$,

where the coefficients $\gamma_{i}^{a}$, for $i=0,1, \ldots, 6$, depend only on $\gamma_{0}$, and are given in Appendix B1.1, and $g_{0}=8\left(92-160 \lambda_{0}+169 \lambda_{0}^{2}-6 \lambda_{0}^{3}+9 \lambda_{0}^{4}+26 \lambda_{0}^{5}\right) y\left(x^{2}+\lambda_{0} x^{2}+\right.$ $\left.\lambda_{0} y^{2}+\lambda_{0} x y-y^{2}\right)\left(x^{2}+y^{2}+\lambda_{1} x^{2} y+\lambda_{1} x y^{2}+\lambda_{1} y^{3}\right)$.

(b) If $\lambda_{2}=\lambda_{4}=0, \lambda_{3}=-\lambda_{1}, \lambda_{6}=\lambda_{1}, \lambda_{5}=2 \lambda_{1}$ and $a=3 / 16$, then system (8) has the Darboux first integral

$H(x, y)=\rho(x, y) \exp \left(-\lambda_{0} \arctan (y / x)\right)|3 / 2+2 \Gamma(x, y)|^{1 / 2}|1 / 2+2 \Gamma(x, y)|^{-3 / 2}$

with

$\Gamma(x, y)=\frac{\lambda_{1}}{14 g_{0}}\left(\gamma_{6}^{b} x^{6}+\gamma_{5}^{b} x^{5} y+\gamma_{4}^{b} x^{4} y^{2}+\gamma_{3}^{b} x^{3} y^{3}+\gamma_{1}^{b} x^{2} y^{4}+\gamma_{1}^{b} x y^{5}+\gamma_{0}^{b} y^{6}\right)$,

$\rho(x, y)=-\left(x^{2}+y^{2}\right)^{3 / 2} /\left(x^{2}+y^{2}+\lambda_{1} x y^{2}+\lambda_{1} x^{2} y+\lambda_{1} y^{3}\right)$,

where $\gamma_{i}^{b}$, for $i=0,1, \ldots, 6$, are given in Appendix B1.2.

(c) If $\lambda_{1}=\lambda_{3}=\lambda_{4}=\lambda_{6}=0, \lambda_{2}=-\lambda_{5}$ and $a=1 / 4$, then system (8) has the Darboux first integral

$H(x, y)=\rho(x, y) \exp \left(-\lambda_{0} \arctan (y / x)+1 /(1+2 \Gamma(x, y))\right)(1+2 \Gamma(x, y))^{-1}$, 
with

$$
\begin{aligned}
& \Gamma(x, y)=\frac{\lambda_{5}}{g_{c 1}}\left(\gamma_{6}^{c 1} x^{6}+\gamma_{5}^{c 1} x^{5} y+\gamma_{4}^{c 1} x^{4} y^{2}+\gamma_{3}^{c 1} x^{3} y^{3}+\gamma_{2}^{c 1} x^{2} y^{4}+\gamma_{1}^{c 1} x y^{5}+\gamma_{0}^{c 1} y^{6}\right), \\
& \rho(x, y)=-\left(x^{2}+y^{2}\right)^{(3 / 2)} /\left(x^{2}+y^{2}+\lambda_{5} x y^{2}+\lambda_{5} x^{2} y\right),
\end{aligned}
$$

where $\gamma_{i}^{c 1}$, for $i=0,1, \ldots, 6$, are given in Appendix B1.3, and $g_{c 1}=16(-300+$ $\left.50 \lambda_{0}-115 \lambda_{0}{ }^{2}+23 \lambda_{0}{ }^{3}-3 \lambda_{0}{ }^{4}+\lambda_{0}{ }^{5}\right)\left(x^{2}+y^{2}+\lambda_{5} x y^{2}+\lambda_{5} x^{2} y\right)\left(x^{3}+2 \lambda_{0} x^{2} y-3 x y^{2}+\right.$ $\left.2 \lambda_{0} x y^{2}+3 y x^{2}-y^{3}\right)$.

(c $\left.c_{2}\right)$ If $\lambda_{2}=3 \lambda_{1}, \lambda_{3}=-\lambda_{1} / 4, \lambda_{4}=-2 \lambda_{1}, \lambda_{5}=\lambda_{1} / 4, \lambda_{6}=\lambda_{1} / 2, a=1 / 4$, then system

(8) has the Darboux first integral given by $H(x, y)$ of $\left(c_{1}\right)$ with

$$
\begin{aligned}
& \Gamma(x, y)=\frac{-\lambda_{1}}{g_{c 2}}\left(\gamma_{6}^{c 2} x^{6}+\gamma_{5}^{c 2} x^{5} y+\gamma_{4}^{c 2} x^{4} y^{2}+\gamma_{3}^{c 2} x^{3} y^{3}+\gamma_{2}^{c 2} x^{2} y^{4}+\gamma_{1}^{c 2} x y^{5}+\gamma_{0}^{c 2} y^{6}\right), \\
& \rho(x, y)=4\left(x^{2}+y^{2}\right)^{3 / 2} /\left(4\left(x^{2}+y^{2}\right)-10 \lambda_{1} x y^{2}-3 \lambda_{1} x^{2} y-8 \lambda_{1} x^{3}+\lambda_{1} y^{3}\right),
\end{aligned}
$$

where $\gamma_{i}^{c 2}$, for $i=0,1, \ldots, 6$, are given in Appendix B1.4, and $g_{c 2}=8(-1149+$ $\left.14183 \lambda_{0}+21744 \lambda_{0}^{2}+4761 \lambda_{0}^{3}+10999 \lambda_{0}^{4}-9702 \lambda_{0}^{5}+1146 \lambda_{0}^{6}\right)\left(-4 x^{2}-4 y^{2}+8 \lambda_{1} x^{3}+\right.$ $\left.10 \lambda_{1} x y^{2}+3 \lambda_{1} y x^{2}-\lambda_{1} y^{3}\right)\left(7 x^{3}+6 \lambda_{0} x^{2} y-9 x y^{2}+16 \lambda_{0} x^{3}+20 \lambda_{0} x y^{2}-2 \lambda_{0} y^{3}-8 y^{3}\right)$.

(d) If $\lambda_{2}=\lambda_{4}=0, \lambda_{3}=-\lambda_{1}, \lambda_{6}=\lambda_{1}, \lambda_{5}=2 \lambda_{1}$ and $a=0$, then system (8) has the Darboux first integral

$$
H(x, y)=\exp \left(\lambda_{0} \arctan \left(\frac{y}{x}\right)\right)\left(\frac{x^{2}+y^{2}+\lambda_{1} x^{2} y+\lambda_{1} y^{3}+\lambda_{1} x y^{2}}{\left(x^{2}+y^{2}\right)^{3 / 2}}+\Gamma(x, y)\right),
$$

with

$$
\begin{aligned}
\Gamma(x, y)=\frac{2 \lambda_{1}}{g_{d}} & \left(\left(-3+9 \lambda_{0}-\lambda_{0}^{2}+\lambda_{0}^{3}\right) x^{3}+\left(3 \lambda_{0}-9 \lambda_{0}{ }^{2}+\lambda_{0}{ }^{3}-\lambda_{0}{ }^{4}\right) x^{2} y\right. \\
& \left.-\left(9-9 \lambda_{0}+8 \lambda_{0}^{2}-\lambda_{0}^{3}+\lambda_{0}^{4}\right) x y^{2}+\left(2 \lambda_{0}-9 \lambda_{0}{ }^{2}-\lambda_{0}^{4}\right) y^{3}\right),
\end{aligned}
$$

where $g_{d}=\left(\lambda_{0}{ }^{4}+10 \lambda_{0}{ }^{2}+9\right)\left(x^{2}+y^{2}\right)^{3 / 2}$.

(e) If $\lambda_{2}=3 \lambda_{1}, \lambda_{3}=-\lambda_{1}, \lambda_{4}=-2 \lambda_{1}, \lambda_{5}=0, \lambda_{6}=\lambda_{1}$ and $a=2 / 9$, then system (8) has the Darboux first integral

$$
H(x, y)=\rho(x, y) \exp \left(-\lambda_{0} \arctan (y / x)\right)(4 / 3+2 \Gamma(x, y))(2 / 3+2 \Gamma(x, y))^{-2}
$$

with

$$
\begin{aligned}
& \Gamma(x, y)=\frac{-\lambda_{1}}{g_{e}}\left(\gamma_{6}^{e} x^{6}+\gamma_{5}^{e} x^{5} y+\gamma_{4}^{e} x^{4} y^{2}+\gamma_{3}^{e} x^{3} y^{3}+\gamma_{2}^{e} x^{2} y^{4}+\gamma_{1}^{e} x y^{5}+\gamma_{0}^{e} y^{6}\right), \\
& \left.\rho(x, y)=\left(x^{2}+y^{2}\right)^{3 / 2} /\left(x^{2}+y^{2}\right)\left(1-2 \lambda_{1} x\right)-\lambda_{1} y\left(x^{2}-y^{2}\right)\right),
\end{aligned}
$$

where $\gamma_{i}^{e}$, for $i=0,1, \ldots, 6$, are given in Appendix B1.5, and $g_{e}=144(-556+$ $\left.123 \lambda_{0}+573 \lambda_{0}{ }^{2}-16 \lambda_{0}^{3}+593 \lambda_{0}{ }^{4}-107 \lambda_{0}{ }^{5}+80 \lambda_{0}{ }^{6}\right)\left(-x^{2}-y^{2}+\lambda_{1} x^{2} y-\lambda_{1} y^{3}+\right.$ $\left.2 \lambda_{1} x^{3}+2 \lambda_{1} x y^{2}\right)\left(2 x^{3}+2 \lambda_{0} x^{2} y+4 \lambda_{0} x^{3}+4 \lambda_{0} x y^{2}-2 \lambda_{0} y^{3}-6 x y^{2}-y x^{2}-y^{3}\right)$.

The following corollary characterizes Darboux first integrals of system (9) belonging to the subclass $\mathcal{J}$.

Corollary 5. System (9) with $\lambda_{0} \neq 0$ belongs to the subclass $\mathcal{J}$ if one of the following statements holds (we note that $\lambda_{9}, \cdots, \lambda_{13}$ can be obtained from (6)). 
(a) If $\lambda_{2}=\lambda_{3}=\lambda_{6}=\lambda_{7}=0, \lambda_{4}=\lambda_{1}, \lambda_{5}=-\lambda_{1}, \lambda_{8}=\lambda_{1}$ and $a=\left(1+\lambda_{0}^{2}\right) /\left(4 \lambda_{0}^{2}\right)$, then system (9) has the Darboux first integral

$$
H(x, y)=\frac{\rho(x, y) \exp \left(-2 \lambda_{0} \arctan (y / x)-\arctan (1+2 \Gamma(x, y))\right)}{\sqrt{\Gamma^{2}(x, y)+\Gamma(x, y)+\left(1+\lambda_{0}^{2}\right) /\left(4 \lambda_{0}^{2}\right)}},
$$

with

$$
\begin{gathered}
\Gamma(x, y)=\frac{-\lambda_{1}}{h_{a}}\left(\eta_{8}^{a} x^{8}+\eta_{7}^{a} x^{7} y+\eta_{6}^{a} x^{6} y^{2}+\eta_{5}^{a} x^{5} y^{3}+\eta_{4}^{a} x^{4} y^{4}+\eta_{3}^{a} x^{3} y^{5}\right. \\
\left.\quad+\eta_{2}^{a} x^{2} y^{6}+\eta_{1}^{a} x y^{7}+\eta_{0}^{a} y^{8}\right), \\
\rho(x, y)=-\left(x^{2}+y^{2}\right)^{2} /\left(-x^{2}-y^{2}+\lambda_{1} x^{4}+\lambda_{1} y^{4}+\lambda_{1} y x^{3}-\lambda_{1} y^{3} x\right),
\end{gathered}
$$

where $\eta_{i}^{a}$, for $i=0,1, \ldots, 8$, are given in Appendix B2.1, and $h_{a}=64 \lambda_{0}{ }^{3}(36+$ $\left.72 \lambda_{0}+23 \lambda_{0}{ }^{2}+46 \lambda_{0}{ }^{3}+4 \lambda_{0}{ }^{4}+8 \lambda_{0}{ }^{5}\right)\left(3 x^{4}-6 x^{2} y^{2}+3 y^{4}-6 x^{3} y+6 x y^{3}+4 \lambda_{0 x^{4}}+\right.$ $\left.4 \lambda_{0} y^{4}+4 \lambda_{0} x^{3} y-4 \lambda_{0} x y^{3}\right)\left(-x^{2}-y^{2}+\lambda_{1} x^{4}+\lambda_{1} y^{4}+\lambda_{1} y x^{3}-\lambda_{1} y^{3} x\right)$.

(b) If $\lambda_{2}=\lambda_{3}=\lambda_{6}=\lambda_{7}=0, \lambda_{4}=-\lambda_{1}, \lambda_{5}=\lambda_{1}, \lambda_{8}=\lambda_{1}$ and $a=1 / 3$, then system (9) has the Darboux first integral

$$
H(x, y)=\frac{\rho(x, y) \exp \left(-2 \lambda_{0} \arctan (y / x)-\arctan (1+2 \Gamma(x, y))\right)}{\sqrt{\Gamma^{2}(x, y)+\Gamma(x, y)+1 / 3}},
$$

with

$$
\begin{gathered}
\Gamma(x, y)=\frac{-\lambda_{1}}{h_{b}}\left(\eta_{8}^{b} x^{8}+\eta_{7}^{b} x^{7} y+\eta_{6}^{b} x^{6} y^{2}+\eta_{5}^{b} x^{5} y^{3}+\eta_{4}^{b} x^{4} y^{4}+\eta_{3}^{b} x^{3} y^{5}\right. \\
\left.+\eta_{2}^{b} x^{2} y^{6}+\eta_{1}^{b} x y^{7}+\eta_{0}^{b} y^{8}\right) \\
\rho(x, y)=\left(x^{2}+y^{2}\right)^{2} /\left(x^{2}+y^{2}+\lambda_{1} x^{4}+\lambda_{1} y^{4}-\lambda_{1} x^{3} y+\lambda_{1} x y^{3}\right),
\end{gathered}
$$

where $\eta_{i}^{b}$, for $i=0,1, \ldots, 8$, are given in Appendix B2.2, and $h_{b}=48 \lambda_{0}(-36+$ $\left.72 \lambda_{0}-23 \lambda_{0}{ }^{2}+46 \lambda_{0}{ }^{3}-4 \lambda_{0}{ }^{4}+8 \lambda_{0}^{5}\right)\left(x^{2}+y^{2}+\lambda_{1} x^{4}+\lambda_{1} y^{4}-\lambda_{1} y x^{3}+\lambda_{1} y^{3} x\right)\left(-3 x^{4}+\right.$ $\left.6 x^{2} y^{2}-3 y^{4}-6 y x^{3}+6 y^{3} x+4 \lambda_{0} x^{4}+4 \lambda_{0} y^{4}-4 \lambda_{0} x^{3} y+4 \lambda_{0} x y^{3}\right)$.

(c) If $\lambda_{2}=\lambda_{6}=\lambda_{7}=\lambda_{8}=0, \lambda_{3}=-\lambda_{1}, \lambda_{4}=-\lambda_{1}, \lambda_{5}=\lambda_{1}$ and $a=1 / 4$, then system (9) has the Darboux first integral

$H(x, y)=\rho(x, y) \exp \left(-2 \lambda_{0} \arctan (y / x)+1 /(1+2 \Gamma(x, y))\right)(1+2 \Gamma(x, y))^{-1}$,

with

$$
\begin{gathered}
\Gamma(x, y)=\frac{-\lambda_{1}}{h_{c}}\left(\eta_{8}^{c} x^{8}+\eta_{7}^{c} x^{7} y+\eta_{6}^{c} x^{6} y^{2}+\eta_{5}^{c} x^{5} y^{3}+\eta_{4}^{c} x^{4} y^{4}+\eta_{3}^{c} x^{3} y^{5}\right. \\
\left.\quad+\eta_{2}^{c} x^{2} y^{6}+\eta_{1}^{c} x y^{7}+\eta_{0}^{c} y^{8}\right) \\
\rho(x, y)=\left(x^{2}+y^{2}\right)^{2} /\left(x^{2}+y^{2}+\lambda_{1} x^{4}+\lambda_{1} y^{4}-\lambda_{1} x^{3} y+\lambda_{1} y^{3}\right),
\end{gathered}
$$

where $\eta_{i}^{c}$, for $i=0,1, \ldots, 8$, are given in Appendix B2.3, and $h_{c}=16 \lambda_{0}(-648-$ $\left.531 \lambda_{0}-1221 \lambda_{0}{ }^{2}-1119 \lambda_{0}{ }^{3}-61 \lambda_{0}{ }^{4}-684 \lambda_{0}{ }^{5}+424 \lambda_{0}{ }^{6}-160 \lambda_{0}{ }^{7}+112 \lambda_{0}{ }^{8}\right)\left(x^{2}+y^{2}+\right.$ $\left.\lambda_{1} x^{4}+\lambda_{1} y^{4}-\lambda_{1} x^{3} y+\lambda_{1} x y^{3}\right)\left(-3 x^{4}+8 x^{2} y^{2}-y^{4}-6 y x^{3}+6 y^{3} x+4 \lambda_{0} x^{4}+4 \lambda_{0} y^{4}-\right.$ $\left.4 \lambda_{0} x^{3} y+4 \lambda_{0} x y^{3}\right)$.

(d) If $\lambda_{2}=\lambda_{7}=0, \lambda_{3}=\lambda_{1}, \lambda_{4}=-\lambda_{1}, \lambda_{5}=\lambda_{1}, \lambda_{6}=\lambda_{1}, \lambda_{8}=-\lambda_{1}$ and $a=2 / 9$, then system (9) has the Darboux first integral $H(x, y)=\rho(x, y) \exp \left(-2 \lambda_{0} \arctan (y / x)\right)(4 / 3+2 \Gamma(x, y))(2 / 3+2 \Gamma(x, y))^{-2}$, 
with

$$
\begin{gathered}
\Gamma(x, y)=\frac{-\lambda_{1}}{h_{d}}\left(\eta_{8}^{d} x^{8}+\eta_{7}^{d} x^{7} y+\eta_{6}^{d} x^{6} y^{2}+\eta_{5}^{d} x^{5} y^{3}+\eta_{4}^{d} x^{4} y^{4}+\eta_{3}^{d} x^{3} y^{5}\right. \\
\left.+\eta_{2}^{d} x^{2} y^{6}+\eta_{1}^{d} x y^{7}+\eta_{0}^{d} y^{8}\right) \\
\rho(x, y)=\left(x^{2}+y^{2}\right)^{2} /\left(x^{2}+y^{2}-2 \lambda_{1} x y^{3}+\lambda_{1} x^{4}+\lambda_{1} y^{4}\right),
\end{gathered}
$$

where $\eta_{i}^{d}$, for $i=0,1, \ldots, 8$, are given in Appendix B2.4, and $h_{d}=288 \lambda_{0}(135-$ $\left.1035 \lambda_{0}+192 \lambda_{0}{ }^{2}-443 \lambda_{0}{ }^{3}-131 \lambda_{0}{ }^{4}+578 \lambda_{0}{ }^{5}-152 \lambda_{0}{ }^{6}+392 \lambda_{0}{ }^{7}-32 \lambda_{0}{ }^{8}+64 \lambda_{0}{ }^{9}\right)\left(x^{2}+\right.$ $\left.y^{2}-2 \lambda_{1} y^{3} x+\lambda_{1} x^{4}+\lambda_{1} y^{4}\right)\left(-x^{4}-5 x^{2} y^{2}+2 y^{4}-3 y x^{3}+3 y^{3} x-4 y^{3} x \lambda_{0}+2 x^{4} \lambda_{0}+2 \lambda_{0} y^{4}\right)$.

(e) If $\lambda_{2}=\lambda_{3}=\lambda_{6}=\lambda_{7}=0, \lambda_{4}=-\lambda_{1}, \lambda_{5}=\lambda_{1}, \lambda_{8}=-\lambda_{1}$ and $a=0$, then system

(9) has the Darboux first integral

$$
\begin{aligned}
& H(x, y)=\exp \left(2 \lambda_{0} \arctan (y / x)\right)\left(\frac{x^{2}+y^{2}+\lambda_{1}\left(x^{4}-x^{3} y-x y^{3}+y^{4}\right)}{\left(x^{2}+y^{2}\right)^{2}}+\Gamma(x, y)\right), \\
& \text { with } \\
& \Gamma(x, y)=\frac{-\lambda_{1}}{2\left(4+5 \lambda_{0}{ }^{2}+\lambda_{0}^{4}\right)\left(x^{2}+y^{2}\right)^{2}}\left(\eta_{4}^{e} x^{4}+\eta_{3}^{e} x^{3} y++\eta_{2}^{e} x^{2} y^{2}+\eta_{1}^{e} x y^{3}+\eta_{0}^{e} y^{4}\right), \\
& \text { where } \eta_{4}^{e}=15-4 \lambda_{0}+19 \lambda_{0}{ }^{2}-\lambda_{0}{ }^{3}+4 \lambda_{0}^{4}, \eta_{3}^{e}=-24+2 \lambda_{0}-22 \lambda_{0}^{2}+2 \lambda_{0}{ }^{3}-4 \lambda_{0}^{4}, \eta_{2}^{e}= \\
& 6+6 \lambda_{0}^{2}, \eta_{1}^{e}=-24-2 \lambda_{0}-22 \lambda_{0}^{2}-2 \lambda_{0}{ }^{3}-4 \lambda_{0}^{4} \text { and } \eta_{0}^{e}=15+4 \lambda_{0}+19 \lambda_{0}{ }^{2}+\lambda_{0}{ }^{3}+4 \lambda_{0}^{4} .
\end{aligned}
$$

The proofs of Corollaries 4 and 5 follow doing tedious computations using statements (a) and (b) of Theorem 2 and computer algebra.

\section{Algebraic limit cycles of Darboux integrable systems}

The existence and determination of limit cycles constitutes a difficult problem in the qualitative theory of ordinary differential equations. The following proposition characterizes a class of polynomial systems having Darboux first integrals and algebraic limit cycles.

Proposition 6. System (1) with $n=2, g(\theta)=0$ and being in the subclass $\mathcal{J}$ can be written in

(16)

$$
\begin{aligned}
& \dot{x}=-y+x\left(\lambda_{0}+\lambda_{1} x^{2}+\lambda_{2} x y+\lambda_{3} y^{2}+\lambda_{4} x^{4}+\lambda_{5} x^{3} y+\lambda_{6} x^{2} y^{2}+\lambda_{7} x y^{3}+\lambda_{8} y^{4}\right), \\
& \dot{y}=x+y\left(\lambda_{0}+\lambda_{1} x^{2}+\lambda_{2} x y+\lambda_{3} y^{2}+\lambda_{4} x^{4}+\lambda_{5} x^{3} y+\lambda_{6} x^{2} y^{2}+\lambda_{7} x y^{3}+\lambda_{8} y^{4}\right),
\end{aligned}
$$

where the coefficients $\lambda_{i}$ 's satisfy one of the conditions given in Corollary 10. Then the following statements hold.

(a) System (16) for $a \leq 1 / 4$ and $a \neq 0$ has the algebraic solutions

$$
f(x, y)=\left\{\begin{array}{l}
\lambda_{0}\left(\lambda_{0}^{2}+1\right)(-1+\sqrt{1-4 a}) / a, \\
\left.\lambda_{0}\left(\lambda_{0}^{2}+1\right)\right)(-1-\sqrt{1-4 a}) / a,
\end{array}\right.
$$


denoted by $\Gamma_{1}$ and $\Gamma_{2}$ respectively, with $f(x, y)=\left(\lambda_{1}+\lambda_{3}-\lambda_{0} \lambda_{2}+2 \lambda_{0}^{2} \lambda_{1}\right) x^{2}+$ $2 \lambda_{0}\left(\lambda_{1}-\lambda_{3}+\lambda_{0} \lambda_{2}\right) x y+\left(\lambda_{1}+\lambda_{3}+\lambda_{0} \lambda_{2}+2 \lambda_{0}^{2} \lambda_{3}\right) y^{2}$, and the Darboux first integral

$$
H(r, \theta)=\frac{r^{2} \exp \left(-2 \lambda_{0} \theta\right)(\sqrt{1-4 a}+1+2 a \Gamma(r, \theta))^{(-1+1 / \sqrt{1-4 a}) / 2}}{(\sqrt{1-4 a}-1-2 a \Gamma(r, \theta))^{(1+1 / \sqrt{1-4 a}) / 2}},
$$

defined on $\mathbb{R}^{2} \backslash \Sigma$, where $\Sigma=\{(0,0)\} \cup\{(r, \theta): 2 a \Gamma(r, \theta)+(1 \pm \sqrt{1-4 a})=0\}$, and $\Gamma(r, \theta)=r^{2}\left(\left(\lambda_{0}^{2} \lambda_{1}-\lambda_{3} \lambda_{0}^{2}-\lambda_{0} \lambda_{2}\right) \cos (2 \theta)+\left(\lambda_{1} \lambda_{0}-\lambda_{0} \lambda_{3}+\lambda_{0}^{2} \lambda_{2}\right) \sin (2 \theta)+\right.$ $\left.\lambda_{0}^{2} \lambda_{1}+\lambda_{1}+\lambda_{0}^{2} \lambda_{3}+\lambda_{3}\right) /\left(2 \lambda_{0}+2 \lambda_{0}^{3}\right)$. Denote $\Delta:=\left(\lambda_{1}+\lambda_{3}\right)^{2}+\lambda_{0}^{2}\left(4 \lambda_{1} \lambda_{3}-\lambda_{2}^{2}\right)$ and $\Lambda:=\lambda_{0}\left(\lambda_{1}+\lambda_{3}\right)$.

(a) If $a=1 / 4, \Delta>0$ and $\Lambda<0$, then system (16) has the limit cycle $\Gamma_{1}=\Gamma_{2}$.

$\left(a_{2}\right)$ If $a<0, \Delta>0$ and $\Lambda<0$ (respectively, $\Lambda>0$ ), then system (16) has the limit cycle $\Gamma_{1}$ (respectively, $\Gamma_{2}$ ).

(a) If $0<a<1 / 4, \Delta>0$ and $\Lambda<0$, then system (16) has the two limit cycles $\Gamma_{1}$ and $\Gamma_{2}$.

(b) If $a=0, \Delta>0$ and $\Lambda<0$, then system (16) has one limit cycle given by $f(x, y)+$ $2\left(\lambda_{0}+\lambda_{0}^{3}\right)=0$, and the Darboux first integral $H(r, \theta)=\exp \left(2 \lambda_{0} \theta\right)(1+\Gamma(r, \theta)) / r^{2}$ defined on $\mathbb{R}^{2} \backslash \Sigma$, where $\Sigma=\{(0,0)\} \cup\{(r, \theta): \Gamma(r, \theta)+1=0\}$.

(c) In the other cases system (16) has no limit cycles.

From Theorems 1 and 2 we can obtain the proof of Proposition 6.

The following result due to Chavarriga et al [1] shows the coexistence of limit cycles and Darboux first integrals of a given system.

Proposition 7. The differential system

$$
\dot{x}=x-y-x\left(x^{2}+y^{2}\right), \quad \dot{y}=x+y-y\left(x^{2}+y^{2}\right),
$$

has the algebraic solution $x^{2}+y^{2}=1$ as a limit cycle. In polar coordinates the function $H(r, \theta)=\left(r^{2}-1\right) \exp (2 \theta) / r^{2}=C$ is a Darboux first integral defined on $\mathbb{R}^{2} \backslash \Sigma$, where $\Sigma=\{(0,0)\} \cup\left\{(x, y): x^{2}+y^{2}=1\right\}$.

We note that this last result can be got from Proposition 6 by taking $a=0, \lambda_{0}=1$, $\lambda_{1}=\lambda_{3}=-1$ and $\lambda_{2}=0$. Some other different examples of this kind can be found in Christopher [4], Dolov [9], and Kooij and Christopher [16]. In what follows we provide some new examples having algebraic limit cycles.

Example 1 System (16) with $a=1 / 4, \lambda_{0}=2, \lambda_{1}=-2, \lambda_{2}=-1 / 2$ and $\lambda_{3}=-3$ is

$$
\begin{aligned}
& \dot{x}=2 x-y+x\left(2 x^{2}+3 y^{2}-8\right)\left(4 x^{2}+x y+6 y^{2}\right) / 16, \\
& \dot{y}=x+2 y+y\left(2 x^{2}+3 y^{2}-8\right)\left(4 x^{2}+x y+6 y^{2}\right) / 16 .
\end{aligned}
$$

It has exactly one limit cycle given by $2 x^{2}+3 y^{2}-4=0$. 
Example 2 System (16) with $a=2 / 9, \lambda_{0}=2, \lambda_{1}=-2, \lambda_{2}=-1 / 2$ and $\lambda_{3}=-3$ is

$$
\begin{aligned}
& \dot{x}=2 x-y+x\left(4 x^{2}+x y+6 y^{2}\right)\left(2 x^{2}+3 y^{2}-9\right) / 18, \\
& \dot{y}=x+2 y+y\left(4 x^{2}+x y+6 y^{2}\right)\left(2 x^{2}+3 y^{2}-9\right) / 18 .
\end{aligned}
$$

It has exactly two limit cycles given by $2 x^{2}+3 y^{2}-3=0$ and $2 x^{2}+3 y^{2}-6=0$.

Example 3 System (16) with $a=0, \lambda_{0}=2, \lambda_{1}=-2, \lambda_{2}=-1 / 2$ and $\lambda_{3}=-3$ is

$$
\dot{x}=2 x-y-x\left(2 x^{2}+\frac{1}{2} x y+3 y^{2}\right), \quad \dot{y}=x+2 y-y\left(2 x^{2}+\frac{1}{2} x y+3 y^{2}\right) .
$$

It has exactly one limit cycle given by $2 x^{2}+3 y^{2}-2=0$.

Proposition 8. System (1) with $n=4, g(\theta)=0$ and being in the subclass $\mathcal{J}$ can be written as

$$
\begin{aligned}
\dot{x}=-y & +x\left(\lambda_{0}+\lambda_{1} x^{4}+\lambda_{2} x^{3} y+\lambda_{3} x^{2} y^{2}+\lambda_{4} x y^{3}+\lambda_{5} y^{4}+\lambda_{6} x^{8}+\lambda_{7} x^{7} y\right. \\
& \left.+\lambda_{8} x^{6} y^{2}+\lambda_{9} x^{5} y^{3}+\lambda_{10} x^{4} y^{4}+\lambda_{11} x^{3} y^{5}+\lambda_{12} x^{2} y^{6}+\lambda_{13} x y^{7}+\lambda_{14} y^{8}\right), \\
\dot{y}=x+ & y\left(\lambda_{0}+\lambda_{1} x^{4}+\lambda_{2} x^{3} y+\lambda_{3} x^{2} y^{2}+\lambda_{4} x y^{3}+\lambda_{5} y^{4}+\lambda_{6} x^{8}+\lambda_{7} x^{7} y\right. \\
& \left.+\lambda_{8} x^{6} y^{2}+\lambda_{9} x^{5} y^{3}+\lambda_{10} x^{4} y^{4}+\lambda_{11} x^{3} y^{5}+\lambda_{12} x^{2} y^{6}+\lambda_{13} x y^{7}+\lambda_{14} y^{8}\right),
\end{aligned}
$$

where the coefficients $\lambda_{i}$ 's satisfy one of the conditions given in Corollary 12. Then the following statements hold.

(a) System (18) for $a \leq 1 / 4$ and $a \neq 0$ has the algebraic solutions

$$
f(x, y)=\left\{\begin{array}{l}
4(-1+\sqrt{1-4 a})\left(4 \lambda_{0}^{2}+1\right)\left(\lambda_{0}^{3}+\lambda_{0}\right) / a, \\
4(-1-\sqrt{1-4 a})\left(4 \lambda_{0}^{2}+1\right)\left(\lambda_{0}^{3}+\lambda_{0}\right) / a,
\end{array}\right.
$$

denoted by $\Gamma_{1}$ and $\Gamma_{2}$ respectively, where $f(x, y)=\gamma_{4} x^{4}+\gamma_{3} x^{3} y+\gamma_{2} x^{2} y^{2}+\gamma_{1} x y^{3}+$ $\gamma_{0} y^{4}$ with the coefficients given by

$$
\begin{aligned}
& \gamma_{4}=\lambda_{3}+3 \lambda_{1}+3 \lambda_{5}-5 \lambda_{0} \lambda_{2}-3 \lambda_{0} \lambda_{4}+32 \lambda_{0}{ }^{2} \lambda_{1}+32 \lambda_{0}{ }^{4} \lambda_{1}+4 \lambda_{0}{ }^{2} \lambda_{3}-8 \lambda_{0}{ }^{3} \lambda_{2}, \\
& \gamma_{3}=4\left(5 \lambda_{1}-\lambda_{3}-3 \lambda_{5}+5 \lambda_{0} \lambda_{2}+3 \lambda_{0} \lambda_{4}+8 \lambda_{0}{ }^{2} \lambda_{1}-4 \lambda_{0}{ }^{2} \lambda_{3}+8 \lambda_{0}{ }^{3} \lambda_{2}\right), \\
& \gamma_{2}=2\left(3 \lambda_{1}+\lambda_{3}+3 \lambda_{5}+3 \lambda_{0} \lambda_{2}-3 \lambda_{0} \lambda_{4}+4 \lambda_{0}{ }^{2} \lambda_{3}\right), \\
& \gamma_{1}=4\left(3 \lambda_{1}+\lambda_{3}-5 \lambda_{5}+3 \lambda_{0} \lambda_{2}+5 \lambda_{0} \lambda_{4}+4 \lambda_{0}{ }^{2} \lambda_{3}-8 \lambda_{0}{ }^{2} \lambda_{5}+8 \lambda_{0}{ }^{3} \lambda_{4}\right), \\
& \gamma_{0}=3 \lambda_{1}+\lambda_{3}+3 \lambda_{5}+3 \lambda_{0} \lambda_{2}+5 \lambda_{0} \lambda_{4}+4 \lambda_{0}{ }^{2} \lambda_{3}+32 \lambda_{0}{ }^{2} \lambda_{5}+8 \lambda_{0}{ }^{3} \lambda_{4}+32 \lambda_{0}{ }^{4} \lambda_{5} .
\end{aligned}
$$

Moreover for suitable choice of the $\lambda_{i}$ 's, $\Gamma_{1}$ and $\Gamma_{2}$ contain ellipses, and consequently the system can have two algebraic limit cycles.

(b) For $a \leq 1 / 4$ and $a \neq 0$ the function in polar coordinates

$$
H(r, \theta)=\frac{r^{4} \exp \left(-4 \lambda_{0} \theta\right)(\sqrt{1-4 a}+1+2 a \gamma(r, \theta))^{(-1+1 / \sqrt{1-4 a}) / 2}}{(\sqrt{1-4 a}-1-2 a \gamma(r, \theta))^{(1+1 / \sqrt{1-4 a}) / 2}},
$$

is a Darboux first integral defined on $\mathbb{R}^{2} \backslash \Sigma$, where $\Sigma=\{(0,0)\} \cup\{(r, \theta): 2 a \gamma(r, \theta)+$ $(1 \pm \sqrt{1-4 a})=0\}$ and $\gamma(r, \theta)=r^{4}\left[c_{4} \cos (4 \theta)+s_{4} \sin (4 \theta)+c_{2} \cos (2 \theta)+s_{2} \sin (2 \theta)+\right.$ 
$\left.c_{0}\right] /\left(8 \lambda_{0}+40 \lambda_{0}^{3}+32 \lambda_{0}^{5}\right)$ with

$$
\begin{aligned}
& c_{4}=-\lambda_{0} \lambda_{2}+\lambda_{0} \lambda_{4}+\lambda_{0}{ }^{2} \lambda_{1}-\lambda_{0}{ }^{2} \lambda_{3}+\lambda_{0}{ }^{2} \lambda_{5}-4 \lambda_{0}{ }^{3} \lambda_{2}+4 \lambda_{0}{ }^{3} \lambda_{4}-4 \lambda_{0}{ }^{4} \lambda_{3} \\
& +4 \lambda_{0}^{4} \lambda_{5}+4 \lambda_{0}{ }^{4} \lambda_{1} \\
& s_{4}=\lambda_{0} \lambda_{1}-\lambda_{0} \lambda_{3}+\lambda_{0} \lambda_{5}+\lambda_{0}{ }^{2} \lambda_{2}-\lambda_{0}{ }^{2} \lambda_{4}+4 \lambda_{0}{ }^{3} \lambda_{1}-4 \lambda_{0}{ }^{3} \lambda_{3}+4 \lambda_{0}{ }^{3} \lambda_{5} \\
& -4 \lambda_{0}{ }^{4} \lambda_{4}+4 \lambda_{0}{ }^{4} \lambda_{2} \\
& c_{2}=-4 \lambda_{0} \lambda_{2}-4 \lambda_{0} \lambda_{4}+16 \lambda_{0}{ }^{2} \lambda_{1}-16 \lambda_{0}{ }^{2} \lambda_{5}-4 \lambda_{0}{ }^{3} \lambda_{2}-4 \lambda_{0}{ }^{3} \lambda_{4}+16 \lambda_{0}{ }^{4} \lambda_{1}-16 \lambda_{0}{ }^{4} \lambda_{5}, \\
& s_{2}=8 \lambda_{0} \lambda_{1}-8 \lambda_{0} \lambda_{5}+8 \lambda_{0}{ }^{2} \lambda_{2}+8 \lambda_{0}{ }^{2} \lambda_{4}+8 \lambda_{0}{ }^{3} \lambda_{1}-8 \lambda_{0}{ }^{3} \lambda_{5}+8 \lambda_{0}{ }^{4} \lambda_{2}+8 \lambda_{0}{ }^{4} \lambda_{4}, \\
& c_{0}=3 \lambda_{1}+\lambda_{3}+3 \lambda_{5}+15 \lambda_{0}{ }^{2} \lambda_{1}+5 \lambda_{0}{ }^{2} \lambda_{3}+15 \lambda_{0}{ }^{2} \lambda_{5}+12 \lambda_{0}{ }^{4} \lambda_{1}+4 \lambda_{0}{ }^{4} \lambda_{3}+12 \lambda_{0}{ }^{4} \lambda_{5} \text {. }
\end{aligned}
$$

(c) If $a=0$, system (18) has the algebraic solution $f(x, y)+8\left(1+4 \lambda_{0}^{2}\right)\left(\lambda_{0}+\lambda_{0}^{3}\right)=0$ with $f(x, y)$ given in $(a)$. Furthermore for suitable choice of the $\lambda_{i}$ 's, the algebraic curve can contain an ellipse and consequently the system can have an algebraic limit cycle.

(d) For $a=0, H(r, \theta)=\exp \left(4 \lambda_{0} \theta\right)(1+\gamma(r, \theta)) / r^{4}$ is a Darboux first integral defined on $\mathbb{R}^{2} \backslash \Sigma$, where $\Sigma=\{(0,0)\} \cup\{(r, \theta): \gamma(r, \theta)+1=0\}$ and $\gamma(r, \theta)$ is given in $(b)$.

The proof of Proposition 8 follows from Theorems 1 and 2.

We note from this last proposition that if $a=1 / 4$ or $a=0$ there exist systems of form (18) belonging to the subclass $\mathcal{J}$ which have one limit cycle; if $a<1 / 4$ and $a \neq 0$ there exist systems of form (18) belonging to the subclass $\mathcal{J}$ which have 1 or 2 limit cycles.

The following examples provide systems (18) having algebraic limit cycles.

Example 4 System (18) with $a=1 / 4, \lambda_{0}=1, \lambda_{1}=\lambda_{2}=\lambda_{4}=-1, \lambda_{3}=-4$ and $\lambda_{5}=-3$, is

$$
\begin{aligned}
& \dot{x}=x-y+\frac{1}{4} x\left(x^{2}+y^{2}\right)\left(x^{2}+x y+3 y^{2}\right)\left(x^{4}+4 x^{2} y^{2}+3 y^{4}-4\right), \\
& \dot{y}=x+y+\frac{1}{4} y\left(x^{2}+y^{2}\right)\left(x^{2}+x y+3 y^{2}\right)\left(x^{4}+4 x^{2} y^{2}+3 y^{4}-4\right) .
\end{aligned}
$$

It has exactly one limit cycle given by $\left(x^{2}+y^{2}\right)\left(x^{2}+3 y^{2}\right)=2$.

Example 5 System (18) with $a=3 / 16, \lambda_{0}=1, \lambda_{1}=\lambda_{2}=\lambda_{4}=-1, \lambda_{3}=-4$ and $\lambda_{5}=-3$ is

$$
\begin{aligned}
& \dot{x}=x-y+\frac{1}{16} x\left(x^{2}+y^{2}\right)\left(x^{2}+x y+3 y^{2}\right)\left(3 x^{4}+12 x^{2} y^{2}+9 y^{4}-16\right), \\
& \dot{y}=x+y+\frac{1}{16} y\left(x^{2}+y^{2}\right)\left(x^{2}+x y+3 y^{2}\right)\left(3 x^{4}+12 x^{2} y^{2}+9 y^{4}-16\right) .
\end{aligned}
$$

It has exactly two limit cycles given by $3\left(x^{2}+y^{2}\right)\left(x^{2}+3 y^{2}\right)=4$ and $\left(x^{2}+y^{2}\right)\left(x^{2}+3 y^{2}\right)=4$.

Example 6 System (18) with $a=0, \lambda_{0}=1, \lambda_{1}=\lambda_{2}=\lambda_{4}=-1, \lambda_{3}=-4$ and $\lambda_{5}=-3$ is

$$
\dot{x}=x-y-x\left(x^{2}+y^{2}\right)\left(x^{2}+x y+3 y^{2}\right), \quad \dot{y}=x+y-y\left(x^{2}+y^{2}\right)\left(x^{2}+x y+3 y^{2}\right) .
$$

It has exactly one limit cycle given by $\left(x^{2}+y^{2}\right)\left(x^{2}+3 y^{2}\right)=1$. 


\section{Conclusions}

We have attempted to study the integrability of a class of planar polynomial differential systems through the Darboux theory of integrability via the Abel differential equations. By using these Abel differential equations we have obtained a large class of Darboux integrable planar polynomial differential systems and we provided the explicit expressions of their first integrals. This shows that the study of the integrability through the Abel differential equations is very useful.

As we know, it is very difficult to detect the existence of first integrals for a given polynomial differential system, and is also difficult to obtain the explicit expression of such a first integral. Applying our general theory to some concrete classes of polynomial differential systems we have covered some known results, and found some new integrable systems. Moreover we provided the concrete expressions of their first integrals.

Another main contents of this paper is on the existence of algebraic limit cycles for the integrable systems. For two classes of integrable polynomial differential systems of degree 5 and 9 , using the inverse integrating factors we have obtained the existence and concrete expressions of their algebraic limit cycles. Such systems having a Darboux first integral and algebraic limit cycles, some are known, but most are new. The known ones were got by several authors using different methods as we mentioned in the context.

In short our methods can be used to obtain rich integrable planar polynomial differential systems, and to get the algebraic limit cycles of these systems.

\section{Appendix A}

System (8) with $g(\theta)=0$ can be written in

$$
\begin{aligned}
& \dot{x}=-y+\lambda_{0} x+x\left(\lambda_{1} x+\lambda_{2} y+\lambda_{3} x^{2}+\lambda_{4} x y+\lambda_{5} y^{2}\right), \\
& \dot{y}=x+\lambda_{0} y+y\left(\lambda_{1} x+\lambda_{2} y+\lambda_{3} x^{2}+\lambda_{4} x y+\lambda_{5} y^{2}\right) .
\end{aligned}
$$

The following result provides the necessary and sufficient conditions for system (19) to be in the subclass $\mathcal{J}$. It was obtained in Corollary 5 of [14] except the case $a=0$.

Corollary 9. System (19) belongs to the subclass $\mathcal{J}$ if and only if one of the following statements holds.

(a) If $\lambda_{1}=\lambda_{2}=0$, then a Darboux first integral is given in Theorem $2(b)$.

(b) If $\lambda_{3}=\lambda_{4}=\lambda_{5}=0$, then a Darboux first integral is given in Theorem $2(b)$.

(c) If $\lambda_{3}=a \lambda_{1}\left(\lambda_{0}-\lambda_{2}\right) /\left(1+\lambda_{0}^{2}\right), \lambda_{4}=a\left(-\lambda_{2}^{2}+\lambda_{1}^{2}+2 \lambda_{1} \lambda_{0} \lambda_{2}\right) /\left(1+\lambda_{0}^{2}\right), \lambda_{5}=a \lambda_{2}\left(\lambda_{1}+\right.$ $\left.\lambda_{0} \lambda_{2}\right) /\left(1+\lambda_{0}^{2}\right)$, then a Darboux first integral is given in Theorem $2(a)$.

Consequently this cubic system has a focus at the origin and a Darboux first integral for $\lambda_{0} \neq 0$; or has a center at the origin and a Darboux first integral for $\lambda_{0}=0$. 
The following result presents the necessary and sufficient conditions for system (16) to be in the subclass $\mathcal{J}$.

Corollary 10. System (16) belongs to the subclass $\mathcal{J}$ if and only if one of the following statements holds.

(a) If $\lambda_{1}=\lambda_{2}=\lambda_{3}=0$, then a Darboux first integral is given in Theorem $2(b)$.

(b) If $\lambda_{4}=\lambda_{5}=\lambda_{6}=\lambda_{7}=\lambda_{8}=0$, then a Darboux first integral is given in Theorem $2(b)$.

(c) If $\lambda_{4}=a \lambda_{1} \mathcal{K}_{1}\left(\lambda_{1}+\lambda_{3}-\lambda_{0} \lambda_{2}+2 \lambda_{1} \lambda_{0}^{2}\right)$,

$\lambda_{5}=a \mathcal{K}_{1}\left(\lambda_{1} \lambda_{2}+\lambda_{2} \lambda_{3}+2 \lambda_{0} \lambda_{1}^{2}-2 \lambda_{0} \lambda_{1} \lambda_{3}-\lambda_{0}{\lambda_{2}}^{2}+4 \lambda_{0}^{2} \lambda_{1} \lambda_{2}\right)$,

$\lambda_{6}=a \mathcal{K}_{1}\left(\lambda_{1}{ }^{2}+2 \lambda_{1} \lambda_{3}+\lambda_{3}{ }^{2}+3 \lambda_{0} \lambda_{1} \lambda_{2}-3 \lambda_{0} \lambda_{2} \lambda_{3}+4 \lambda_{0}{ }^{2} \lambda_{1} \lambda_{3}+2 \lambda_{0}{ }^{2} \lambda_{2}{ }^{2}\right)$,

$\lambda_{7}=a \mathcal{K}_{1}\left(\lambda_{1} \lambda_{2}+\lambda_{2} \lambda_{3}+2 \lambda_{0} \lambda_{1} \lambda_{3}+\lambda_{0}{\lambda_{2}}^{2}-2 \lambda_{0} \lambda_{3}^{2}+4 \lambda_{0}{ }^{2} \lambda_{3} \lambda_{2}\right)$,

$\lambda_{8}=a \lambda_{3} \mathcal{K}_{1}\left(\lambda_{1}+\lambda_{3}+\lambda_{0} \lambda_{2}+2 \lambda_{0}^{2} \lambda_{3}\right), \quad \mathcal{K}_{1}=1 /\left(2 \lambda_{0}+2 \lambda_{0}^{3}\right)$,

then a Darboux first integral is given in Theorem $2(a)$.

Consequently this quintic system has a focus at the origin and a Darboux first integral for $\lambda_{0} \neq 0$; or has a center at the origin and a Darboux first integral for $\lambda_{0}=0$.

Systems (10) with $g(\theta)=0$ can be written in

$$
\begin{aligned}
\dot{x}=-y & +x\left(\lambda_{0}+\lambda_{1} x^{3}+\lambda_{2} x^{2} y+\lambda_{3} x y^{2}+\lambda_{4} y^{3}+\lambda_{5} x^{6}\right. \\
& \left.+\lambda_{6} x^{5} y+\lambda_{7} x^{4} y^{2}+\lambda_{8} x^{3} y^{3}+\lambda_{9} x^{2} y^{4}+\lambda_{10} x y^{5}+\lambda_{11} y^{6}\right), \\
\dot{y}=x+ & y\left(\lambda_{0}+\lambda_{1} x^{3}+\lambda_{2} x^{2} y+\lambda_{3} x y^{2}+\lambda_{4} y^{3}+\lambda_{5} x^{6}+\lambda_{6} x^{5} y\right. \\
& \left.+\lambda_{6} x^{5} y+\lambda_{7} x^{4} y^{2}+\lambda_{8} x^{3} y^{3}+\lambda_{9} x^{2} y^{4}+\lambda_{10} x y^{5}+\lambda_{11} y^{6}\right),
\end{aligned}
$$

where $\lambda_{i}$, for $i=0, \cdots, 11$ are arbitrary constants. Then we have

Corollary 11. System (20) belongs to the subclass $\mathcal{J}$ if and only if one of the following statements holds.

(a) If $\lambda_{1}=\lambda_{2}=\lambda_{3}=\lambda_{4}=0$, then system (20) has the Darboux first integral given in Theorem $2(b)$.

(b) If $\lambda_{5}=\lambda_{6}=\lambda_{7}=\lambda_{8}=\lambda_{9}=\lambda_{10}=\lambda_{11}=0$, then system (20) has the Darboux first integral given in Theorem $2(b)$.

(c) If

$$
\begin{aligned}
& \lambda_{5}=\lambda_{1} \mathcal{K}_{2}\left(-\lambda_{2}-2 \lambda_{4}+7 \lambda_{0} \lambda_{1}+2 \lambda_{0} \lambda_{3}-3 \lambda_{0}{ }^{2} \lambda_{2}+9 \lambda_{0}{ }^{3} \lambda_{1}\right), \\
& \lambda_{6}=\mathcal{K}_{2}\left(3 \lambda_{1}{ }^{2}-\lambda_{2}{ }^{2}-2 \lambda_{2} \lambda_{4}+10 \lambda_{0} \lambda_{1} \lambda_{2}+6 \lambda_{0} \lambda_{1} \lambda_{4}+2 \lambda_{0} \lambda_{2} \lambda_{3}+9 \lambda_{0}{ }^{2} \lambda_{1}{ }^{2}\right. \\
& \left.\quad-6 \lambda_{0}{ }^{2} \lambda_{1} \lambda_{3}-3 \lambda_{0}{ }^{2} \lambda_{2}{ }^{2}+18 \lambda_{0}{ }^{3} \lambda_{1} \lambda_{2}\right), \\
& \lambda_{7}=\mathcal{K}_{2}\left(3 \lambda_{1} \lambda_{2}-3 \lambda_{1} \lambda_{4}-\lambda_{2} \lambda_{3}-2 \lambda_{3} \lambda_{4}+6 \lambda_{0} \lambda_{1}{ }^{2}+10 \lambda_{0} \lambda_{1} \lambda_{3}+3 \lambda_{0} \lambda_{2}{ }^{2}+6 \lambda_{0} \lambda_{2} \lambda_{4}\right. \\
& \left.\quad+15 \lambda_{0}{ }^{2} \lambda_{1} \lambda_{2}-9 \lambda_{0}{ }^{2} \lambda_{1} \lambda_{4}+2 \lambda_{0} \lambda_{3}{ }^{2}-9 \lambda_{0}{ }^{2} \lambda_{2} \lambda_{3}+18 \lambda_{0}{ }^{3} \lambda_{1} \lambda_{3}+9 \lambda_{0}{ }^{3} \lambda_{2}{ }^{2}\right),
\end{aligned}
$$




$$
\begin{gathered}
\lambda_{8}=2 \mathcal{K}_{2}\left(\lambda_{1}^{2}+2 \lambda_{1} \lambda_{3}-2 \lambda_{2} \lambda_{4}-\lambda_{4}{ }^{2}+4 \lambda_{0} \lambda_{1} \lambda_{2}+7 \lambda_{0} \lambda_{1} \lambda_{4}+3 \lambda_{0} \lambda_{2} \lambda_{3}+4 \lambda_{0} \lambda_{3} \lambda_{4}\right. \\
\left.+6 \lambda_{0}{ }^{2} \lambda_{1} \lambda_{3}-6 \lambda_{0}{ }^{2} \lambda_{2} \lambda_{4}-3 \lambda_{0}{ }^{2} \lambda_{3}{ }^{2}+3 \lambda_{0}{ }^{2} \lambda_{2}{ }^{2}+9 \lambda_{0}{ }^{3} \lambda_{1} \lambda_{4}+9 \lambda_{0}{ }^{3} \lambda_{2} \lambda_{3}\right), \\
\lambda_{9}=\mathcal{K}_{2}\left(2 \lambda_{1} \lambda_{2}+3 \lambda_{1} \lambda_{4}+\lambda_{2} \lambda_{3}-3 \lambda_{3} \lambda_{4}+6 \lambda_{0} \lambda_{1} \lambda_{3}+2 \lambda_{0} \lambda_{2}{ }^{2}+3 \lambda_{0} \lambda_{3}{ }^{2}+10 \lambda_{0} \lambda_{2} \lambda_{4}\right. \\
\left.+6 \lambda_{0} \lambda_{4}{ }^{2}+9 \lambda_{0}{ }^{2} \lambda_{1} \lambda_{4}+9 \lambda_{0}{ }^{2} \lambda_{2} \lambda_{3}-15 \lambda_{0}{ }^{2} \lambda_{3} \lambda_{4}+18 \lambda_{0}{ }^{3} \lambda_{2} \lambda_{4}+9 \lambda_{0}{ }^{3} \lambda_{3}{ }^{2}\right), \\
\lambda_{10}=\mathcal{K}_{2}\left(2 \lambda_{1} \lambda_{3}+\lambda_{3}{ }^{2}-3 \lambda_{4}{ }^{2}+6 \lambda_{0} \lambda_{1} \lambda_{4}+2 \lambda_{0} \lambda_{2} \lambda_{3}+10 \lambda_{0} \lambda_{3} \lambda_{4}+3 \lambda_{0}{ }^{2} \lambda_{3}{ }^{2}\right. \\
\left.-9 \lambda_{0}{ }^{2} \lambda_{4}{ }^{2}+6 \lambda_{0}{ }^{2} \lambda_{2} \lambda_{4}+18 \lambda_{0}{ }^{3} \lambda_{3} \lambda_{4}\right), \\
\lambda_{11}=\lambda_{4} \mathcal{K}_{2}\left(9 \lambda_{0}{ }^{3} \lambda_{4}+3 \lambda_{0}{ }^{2} \lambda_{3}+7 \lambda_{4} \lambda_{0}+2 \lambda_{2} \lambda_{0}+2 \lambda_{1}+\lambda_{3}\right),
\end{gathered}
$$

with $\mathcal{K}_{2}=a /\left(1+10 \lambda_{0}^{2}+9 \lambda_{0}^{4}\right)$, then system (20) has the Darboux first integral given in Theorem $2(a)$.

Consequently these systems have a focus at the origin and a Darboux first integral for $\lambda_{0} \neq 0$; or have a center at the origin and a Darboux first integral for $\lambda_{0}=0$.

The following corollary characterizes the Darboux first integrals of polynomial systems (18) of degree 9 belonging to the subclass $\mathcal{J}$.

Corollary 12. System (18) belongs to the subclass $\mathcal{J}$ if and only if one of the following statements holds.

(a) If $\lambda_{1}=\lambda_{2}=\lambda_{3}=\lambda_{4}=\lambda_{5}=0$, then (18) has the Darboux first integral given in Theorem $2(b)$.

(b) If $\lambda_{6}=\lambda_{7}=\lambda_{8}=\lambda_{9}=\lambda_{10}=\lambda_{11}=\lambda_{12}=\lambda_{13}=\lambda_{14}=0$, then (18) has the Darboux first integral given in Theorem $2(b)$.

(c) If

$$
\begin{aligned}
& \lambda_{6}=\lambda_{1} \mathcal{N}\left(3 \lambda_{1}+\lambda_{3}+3 \lambda_{5}-5 \lambda_{0} \lambda_{2}-3 \lambda_{0} \lambda_{4}+32 \lambda_{0}{ }^{2} \lambda_{1}+4 \lambda_{0}{ }^{2} \lambda_{3}-8 \lambda_{0}{ }^{3} \lambda_{2}+32 \lambda_{0}{ }^{4} \lambda_{1}\right) \\
& \lambda_{7}=\mathcal{N}(\left(3 \lambda_{1} \lambda_{2}+\lambda_{2} \lambda_{3}+3 \lambda_{2} \lambda_{5}+20 \lambda_{0} \lambda_{1}{ }^{2}-4 \lambda_{0} \lambda_{1} \lambda_{3}-12 \lambda_{0} \lambda_{1} \lambda_{5}-5 \lambda_{0} \lambda_{2}{ }^{2}\right. \\
&-3 \lambda_{0} \lambda_{2} \lambda_{4}+52 \lambda_{0}{ }^{2} \lambda_{1} \lambda_{2}+12 \lambda_{0}{ }^{2} \lambda_{1} \lambda_{4}+4 \lambda_{0}{ }^{2} \lambda_{2} \lambda_{3}+32 \lambda_{0}{ }^{3} \lambda_{1}{ }^{2} \\
&\left.-16 \lambda_{0}{ }^{3} \lambda_{1} \lambda_{3}-8 \lambda_{0}{ }^{3} \lambda_{2}{ }^{2}+64 \lambda_{0}{ }^{4} \lambda_{1} \lambda_{2}\right), \\
& \lambda_{8}=\mathcal{N}\left(6 \lambda_{1}{ }^{2}+5 \lambda_{1} \lambda_{3}+6 \lambda_{1} \lambda_{5}+\lambda_{3}{ }^{2}+3 \lambda_{3} \lambda_{5}+26 \lambda_{0} \lambda_{1} \lambda_{2}-6 \lambda_{0} \lambda_{1} \lambda_{4}-9 \lambda_{0} \lambda_{2} \lambda_{3}\right. \\
& \quad-12 \lambda_{0} \lambda_{2} \lambda_{5}-3 \lambda_{0} \lambda_{3} \lambda_{4}+24 \lambda_{0}{ }^{2} \lambda_{1}{ }^{2}+48 \lambda_{0}{ }^{2} \lambda_{1} \lambda_{3}+24 \lambda_{0}{ }^{2} \lambda_{1} \lambda_{5} \\
&+ \\
&+20 \lambda_{0}{ }^{2} \lambda_{2}{ }^{2}+12 \lambda_{0}{ }^{2} \lambda_{2} \lambda_{4}+4 \lambda_{0}{ }^{2} \lambda_{3}{ }^{2}+56 \lambda_{0}{ }^{3} \lambda_{1} \lambda_{2}-24 \lambda_{0}{ }^{3} \lambda_{1} \lambda_{4} \\
&\left.\quad+64 \lambda_{0}{ }^{4} \lambda_{1} \lambda_{3}+32 \lambda_{0}{ }^{4} \lambda_{2}{ }^{2}-24 \lambda_{0}{ }^{3} \lambda_{2} \lambda_{3}\right), \\
& \lambda_{9}=\mathcal{N}\left(6 \lambda_{1} \lambda_{2}+3 \lambda_{1} \lambda_{4}+2 \lambda_{2} \lambda_{3}+6 \lambda_{2} \lambda_{5}+\lambda_{3} \lambda_{4}+3 \lambda_{4} \lambda_{5}+12 \lambda_{0} \lambda_{1}{ }^{2}+24 \lambda_{0} \lambda_{1} \lambda_{3}\right. \\
& \quad-20 \lambda_{0} \lambda_{1} \lambda_{5}+6 \lambda_{0} \lambda_{2}{ }^{2}-11 \lambda_{0} \lambda_{2} \lambda_{4}-4 \lambda_{0} \lambda_{3}{ }^{2}-12 \lambda_{0} \lambda_{3} \lambda_{5}-3 \lambda_{0} \lambda_{4}{ }^{2}+36 \lambda_{0}{ }^{2} \lambda_{1} \lambda_{2} \\
&+ \\
&+52 \lambda_{0}{ }^{2} \lambda_{1} \lambda_{4}+36 \lambda_{0}{ }^{2} \lambda_{2} \lambda_{3}+24 \lambda_{0}{ }^{2} \lambda_{2} \lambda_{5}+16 \lambda_{0}{ }^{2} \lambda_{3} \lambda_{4}+48 \lambda_{0}{ }^{3} \lambda_{1} \lambda_{3}-32 \lambda_{0}{ }^{3} \lambda_{1} \lambda_{5} \\
&+\left.24 \lambda_{0}{ }^{3} \lambda_{2}{ }^{2}-32 \lambda_{0}{ }^{3} \lambda_{2} \lambda_{4}-16 \lambda_{0}{ }^{3} \lambda_{3}{ }^{2}+64 \lambda_{0}{ }^{4} \lambda_{1} \lambda_{4}+64 \lambda_{0}{ }^{4} \lambda_{2} \lambda_{3}\right),
\end{aligned}
$$




$$
\begin{aligned}
& \lambda_{10}=\mathcal{N}\left(3 \lambda_{1}^{2}+7 \lambda_{1} \lambda_{3}+6 \lambda_{1} \lambda_{5}+2 \lambda_{3}^{2}+7 \lambda_{3} \lambda_{5}+3 \lambda_{5}^{2}+15 \lambda_{0} \lambda_{1} \lambda_{2}+25 \lambda_{0} \lambda_{1} \lambda_{4}\right. \\
& +10 \lambda_{0} \lambda_{2} \lambda_{3}-25 \lambda_{0} \lambda_{2} \lambda_{5}-10 \lambda_{0} \lambda_{3} \lambda_{4}-15 \lambda_{0} \lambda_{4} \lambda_{5}+28 \lambda_{0}{ }^{2} \lambda_{1} \lambda_{3}+64 \lambda_{0}^{2} \lambda_{1} \lambda_{5} \\
& +12 \lambda_{0}^{2} \lambda_{2}^{2}+40 \lambda_{0}{ }^{2} \lambda_{2} \lambda_{4}+16 \lambda_{0}^{2} \lambda_{3}^{2}+28 \lambda_{0}{ }^{2} \lambda_{3} \lambda_{5}+12 \lambda_{0}{ }^{2} \lambda_{4}{ }^{2}+40 \lambda_{0}{ }^{3} \lambda_{1} \lambda_{4} \\
& \left.+40 \lambda_{0}{ }^{3} \lambda_{2} \lambda_{3}-40 \lambda_{0}{ }^{3} \lambda_{2} \lambda_{5}-40 \lambda_{0}{ }^{3} \lambda_{3} \lambda_{4}+64 \lambda_{0}{ }^{4} \lambda_{1} \lambda_{5}+64 \lambda_{0}{ }^{4} \lambda_{2} \lambda_{4}+32 \lambda_{0}{ }^{4} \lambda_{3}{ }^{2}\right), \\
& \lambda_{11}=\mathcal{N}\left(3 \lambda_{1} \lambda_{2}+6 \lambda_{1} \lambda_{4}+\lambda_{2} \lambda_{3}+3 \lambda_{2} \lambda_{5}+2 \lambda_{3} \lambda_{4}+6 \lambda_{4} \lambda_{5}+20 \lambda_{0} \lambda_{1} \lambda_{5}+12 \lambda_{0} \lambda_{1} \lambda_{3}\right. \\
& +3 \lambda_{0}{\lambda_{2}}^{2}+11 \lambda_{0} \lambda_{2} \lambda_{4}+4 \lambda_{0} \lambda_{3}{ }^{2}-24 \lambda_{0} \lambda_{3} \lambda_{5}-6 \lambda_{0} \lambda_{4}{ }^{2}-12 \lambda_{0} \lambda_{5}{ }^{2}+24 \lambda_{0}{ }^{2} \lambda_{1} \lambda_{4} \\
& +16 \lambda_{0}{ }^{2} \lambda_{2} \lambda_{3}+52 \lambda_{0}{ }^{2} \lambda_{2} \lambda_{5}+36 \lambda_{0}{ }^{2} \lambda_{3} \lambda_{4}+36 \lambda_{0}{ }^{2} \lambda_{4} \lambda_{5}+16 \lambda_{0}{ }^{3} \lambda_{3}{ }^{2}+32 \lambda_{0}{ }^{3} \lambda_{1} \lambda_{5} \\
& \left.+32 \lambda_{0}{ }^{3} \lambda_{2} \lambda_{4}-48 \lambda_{0}{ }^{3} \lambda_{3} \lambda_{5}-24 \lambda_{0}{ }^{3} \lambda_{4}{ }^{2}+64 \lambda_{0}{ }^{4} \lambda_{2} \lambda_{5}+64 \lambda_{0}{ }^{4} \lambda_{3} \lambda_{4}\right), \\
& \lambda_{12}=\mathcal{N}\left(3 \lambda_{1} \lambda_{3}+6 \lambda_{1} \lambda_{5}+\lambda_{3}^{2}+5 \lambda_{3} \lambda_{5}+6 \lambda_{5}^{2}+12 \lambda_{0} \lambda_{1} \lambda_{4}+3 \lambda_{0} \lambda_{2} \lambda_{3}+6 \lambda_{0} \lambda_{2} \lambda_{5}\right. \\
& +9 \lambda_{0} \lambda_{3} \lambda_{4}-26 \lambda_{0} \lambda_{4} \lambda_{5}+24 \lambda_{0}{ }^{2} \lambda_{1} \lambda_{5}+12 \lambda_{0}{ }^{2} \lambda_{2} \lambda_{4}+4 \lambda_{0}{ }^{2} \lambda_{3}{ }^{2}+48 \lambda_{0}{ }^{2} \lambda_{3} \lambda_{5} \\
& +20 \lambda_{0}{ }^{2} \lambda_{4}{ }^{2}+24 \lambda_{0}{ }^{2} \lambda_{5}{ }^{2}+24 \lambda_{0}{ }^{3} \lambda_{2} \lambda_{5}+24 \lambda_{0}{ }^{3} \lambda_{3} \lambda_{4}-56 \lambda_{0}{ }^{3} \lambda_{4} \lambda_{5} \\
& \left.+64 \lambda_{0}{ }^{4} \lambda_{3} \lambda_{5}+32 \lambda_{0}{ }^{4} \lambda_{4}{ }^{2}\right), \\
& \lambda_{13}=\mathcal{N}\left(3 \lambda_{1} \lambda_{4}+\lambda_{3} \lambda_{4}+3 \lambda_{4} \lambda_{5}+12 \lambda_{0} \lambda_{1} \lambda_{5}+3 \lambda_{0} \lambda_{2} \lambda_{4}+4 \lambda_{0} \lambda_{3} \lambda_{5}+5 \lambda_{0} \lambda_{4}{ }^{2}\right. \\
& -20 \lambda_{0} \lambda_{5}{ }^{2}+12 \lambda_{0}{ }^{2} \lambda_{2} \lambda_{5}+4 \lambda_{0}{ }^{2} \lambda_{3} \lambda_{4}+8 \lambda_{0}{ }^{3} \lambda_{4}{ }^{2}+52 \lambda_{0}{ }^{2} \lambda_{4} \lambda_{5}-32 \lambda_{0}{ }^{3} \lambda_{5}{ }^{2} \\
& \left.+16 \lambda_{0}{ }^{3} \lambda_{3} \lambda_{5}+64 \lambda_{4} \lambda_{5} \lambda_{0}{ }^{4}\right), \\
& \lambda_{14}=\mathcal{N} \lambda_{5}\left(3 \lambda_{1}+\lambda_{3}+3 \lambda_{5}+3 \lambda_{0} \lambda_{2}+5 \lambda_{0} \lambda_{4}+4 \lambda_{0}{ }^{2} \lambda_{3}+8 \lambda_{0}{ }^{3} \lambda_{4}+32 \lambda_{0}{ }^{2} \lambda_{5}+32 \lambda_{0}{ }^{4} \lambda_{5}\right), \\
& \text { where } \mathcal{N}=a /\left(8 \lambda_{0}\left(1+5 \lambda_{0}^{2}+4 \lambda_{0}^{4}\right)\right) \text {, then system (18) has the Darboux first integrals } \\
& \text { given in Theorem } 2(a) \text {. }
\end{aligned}
$$

Consequently this system has a focus at the origin and a Darboux first integral for $\lambda_{0} \neq 0$; or has a center at the origin and a Darboux first integral for $\lambda_{0}=0$.

The proof of Corollaries 9, 10, 11 and 12 follows doing tedious computations and using the condition (6), Theorem 2 and computer algebra.

\section{Appendix B}

\section{B1.1}

$$
\begin{aligned}
\gamma_{6}^{a}= & -272+800 \lambda_{0}-1113 \lambda_{0}^{2}+462 \lambda_{0}^{3}-206 \lambda_{0}^{4}-156 \lambda_{0}^{5}+272 \lambda_{0}^{6}, \\
\gamma_{5}^{a}=- & 240 \lambda_{0}+996 \lambda_{0}^{2}-1255 \lambda_{0}{ }^{3}+108 \lambda_{0}{ }^{4}+958 \lambda_{0}{ }^{5}+100 \lambda_{0}{ }^{6}-36 \lambda_{0}{ }^{7}-104 \lambda_{0}{ }^{8}, \\
\gamma_{4}^{a}=- & 544+920 \lambda_{0}+191 \lambda_{0}{ }^{2}-2986 \lambda_{0}{ }^{3}+2203 \lambda_{0}{ }^{4}-1448 \lambda_{0}{ }^{5}+1080 \lambda_{0}{ }^{6} \\
& -60 \lambda_{0}{ }^{7}-68 \lambda_{0}^{8}+104 \lambda_{0}{ }^{9}, \\
\gamma_{3}^{a}=- & 480 \lambda_{0}+1992 \lambda_{0}{ }^{2}-2510 \lambda_{0}{ }^{3}+952 \lambda_{0}{ }^{4}+636 \lambda_{0}{ }^{5}+1552 \lambda_{0}{ }^{6} \\
& -120 \lambda_{0}{ }^{7}-136 \lambda_{0}^{8}+208 \lambda_{0}{ }^{9}, \\
\gamma_{2}^{a}=- & 272-560 \lambda_{0}+3721 \lambda_{0}{ }^{2}-7358 \lambda_{0}{ }^{3}+5392 \lambda_{0}{ }^{4}-3068 \lambda_{0}{ }^{5}+2020 \lambda_{0}{ }^{6} \\
& -144 \lambda_{0}^{7}-100 \lambda_{0}^{8}+312 \lambda_{0}^{9},
\end{aligned}
$$




$$
\begin{aligned}
& \gamma_{1}^{a}=-240 \lambda_{0}+996 \lambda_{0}^{2}-1623 \lambda_{0}^{3}+1484 \lambda_{0}^{4}-998 \lambda_{0}^{5}+1476 \lambda_{0}^{6}-120 \lambda_{0}^{7} \\
& \quad-136 \lambda_{0}^{8}+208 \lambda_{0}^{9}, \\
& \gamma_{0}^{a}=-680 \lambda_{0}+2417 \lambda_{0}^{2}-3910 \lambda_{0}^{3}+2615 \lambda_{0}^{4}-1136 \lambda_{0}^{5}+536 \lambda_{0}^{6} \\
& \quad-60 \lambda_{0}^{7}-68 \lambda_{0}^{8}+104 \lambda_{0}^{9} .
\end{aligned}
$$

B1.2

$$
\begin{aligned}
\gamma_{6}^{b} & =-424 \lambda_{0}+864 \lambda_{0}{ }^{2}-829 \lambda_{0}^{3}-566 \lambda_{0}{ }^{4}+852 \lambda_{0}{ }^{5}+26 \lambda_{0}{ }^{6}, \\
\gamma_{5}^{b} & =-1472+5504 \lambda_{0}-3544 \lambda_{0}{ }^{2}-2204 \lambda_{0}{ }^{3}+6473 \lambda_{0}{ }^{4}+1310 \lambda_{0}{ }^{5}+416 \lambda_{0}{ }^{6}, \\
\gamma_{4}^{b} & =-664 \lambda_{0}+2648 \lambda_{0}{ }^{2}-4421 \lambda_{0}^{3}+1126 \lambda_{0}{ }^{4}+2223 \lambda_{0}{ }^{5}-1144 \lambda_{0}{ }^{6}, \\
\gamma_{3}^{b} & =-2944-768 \lambda_{0}+13392 \lambda_{0}{ }^{2}-26040 \lambda_{0}{ }^{3}+13714 \lambda_{0}{ }^{4}+1468 \lambda_{0}{ }^{5}-2496 \lambda_{0}{ }^{6}, \\
\gamma_{2}^{b} & =-5944 \lambda_{0}+12944 \lambda_{0}{ }^{2}-17171 \lambda_{0}^{3}+4334 \lambda_{0}^{4}+1314 \lambda_{0}{ }^{5}-4030 \lambda_{0}{ }^{6}, \\
\gamma_{1}^{b} & =4416-16512 \lambda_{0}+27752 \lambda_{0}{ }^{2}-24220 \lambda_{0}{ }^{3}+7817 \lambda_{0}{ }^{4}+1822 \lambda_{0}{ }^{5}-2912 \lambda_{0}{ }^{6}, \\
\gamma_{0}^{b} & =184 \lambda_{0}+920 \lambda_{0}^{2}-2763 \lambda_{0}^{3}+2258 \lambda_{0}^{4}+519 \lambda_{0}^{5}-1196 \lambda_{0}^{6} .
\end{aligned}
$$

B1.3

$$
\begin{aligned}
& \gamma_{6}^{c 1}=-150-555 \lambda_{0}+140 \lambda_{0}^{2}-35 \lambda_{0}^{3}+8 \lambda_{0}^{4}, \\
& \gamma_{5}^{c 1}=-2040 \lambda_{0}+244 \lambda_{0}{ }^{2}-6 \lambda_{0}{ }^{3}+18 \lambda_{0}{ }^{4}, \\
& \gamma_{4}^{c 1}=4950+2295 \lambda_{0}+1460 \lambda_{0}{ }^{2}+1377 \lambda_{0}{ }^{3}-302 \lambda_{0}{ }^{4}+32 \lambda_{0}{ }^{5}-16 \lambda_{0}{ }^{6}, \\
& \gamma_{3}^{c 1}=5520 \lambda_{0}-1112 \lambda_{0}{ }^{2}+3668 \lambda_{0}{ }^{3}-700 \lambda_{0}{ }^{4}+96 \lambda_{0}{ }^{5}-32 \lambda_{0}{ }^{6} \text {, } \\
& \gamma_{2}^{c 1}=-4050+3855 \lambda_{0}-2220 \lambda_{0}{ }^{2}+2123 \lambda_{0}{ }^{3}-404 \lambda_{0}{ }^{4}+64 \lambda_{0}{ }^{5}-16 \lambda_{0}{ }^{6} \text {, } \\
& \gamma_{1}^{c 1}=-2040 \lambda_{0}+244 \lambda_{0}{ }^{2}-6 \lambda_{0}{ }^{3}+18 \lambda_{0}^{4} \text {, } \\
& \gamma_{0}^{c 1}=450-595 \lambda_{0}+140 \lambda_{0}{ }^{2}-25 \lambda_{0}{ }^{3}+2 \lambda_{0}{ }^{4} \text {. }
\end{aligned}
$$

B1.4

$$
\begin{aligned}
\gamma_{6}^{c 2}= & 60240+1445271 \lambda_{0}+5256826 \lambda_{0}{ }^{2}+8199055 \lambda_{0}{ }^{3} \\
& +8523988 \lambda_{0}{ }^{4}+3010256 \lambda_{0}{ }^{5}-4674048 \lambda_{0}{ }^{6}+586752 \lambda_{0}{ }^{7}, \\
\gamma_{5}^{c 2}= & -157140+228996 \lambda_{0}+6175852 \lambda_{0}{ }^{2}+11842296 \lambda_{0}{ }^{3}+9985168 \lambda_{0}{ }^{4} \\
& +483048 \lambda_{0}{ }^{5}-3322176 \lambda_{0}{ }^{6}+440064 \lambda_{0}{ }^{7}, \\
\gamma_{4}^{c 2}= & 107136+980181 \lambda_{0}+10252950 \lambda_{0}{ }^{2}+18676157 \lambda_{0}{ }^{3}+18868064 \lambda_{0}{ }^{4} \\
& +9743624 \lambda_{0}^{5}-12640368 \lambda_{0}{ }^{6}+1549392 \lambda_{0}{ }^{7}, \\
\gamma_{3}^{c 2}= & 16632-3148728 \lambda_{0}+189304 \lambda_{0}{ }^{2}+13267920 \lambda_{0}{ }^{3}+14822048 \lambda_{0}{ }^{4} \\
& -815312 \lambda_{0}{ }^{5}-2938368 \lambda_{0}{ }^{6}+403392 \lambda_{0}{ }^{7}, \\
\gamma_{2}^{c 2}= & 88704-2578251 \lambda_{0}-2208418 \lambda_{0}{ }^{2}+3481117 \lambda_{0}{ }^{3}+9655636 \lambda_{0}{ }^{4} \\
& +6346592 \lambda_{0}{ }^{5}-7277568 \lambda_{0}{ }^{6}+861792 \lambda_{0}{ }^{7},
\end{aligned}
$$




$$
\begin{aligned}
\gamma_{1}^{c 2}= & 63468-1869084 \lambda_{0}-5714548 \lambda_{0}{ }^{2}-900552 \lambda_{0}{ }^{3}+5283376 \lambda_{0}{ }^{4} \\
& -3637624 \lambda_{0}{ }^{5}+1735680 \lambda_{0}{ }^{6}-183360 \lambda_{0}{ }^{7}, \\
\gamma_{0}^{c 2}= & -105264-408041 \lambda_{0}-3059742 \lambda_{0}{ }^{2}-4299153 \lambda_{0}{ }^{3}+1176488 \lambda_{0}{ }^{4} \\
& -572728 \lambda_{0}{ }^{5}-95952 \lambda_{0}{ }^{6}+9168 \lambda_{0}{ }^{7} .
\end{aligned}
$$

\section{B1.5}

$$
\begin{gathered}
\gamma_{6}^{e}=-26324-66459 \lambda_{0}-35836 \lambda_{0}{ }^{2}+17234 \lambda_{0}{ }^{3}+236500 \lambda_{0}{ }^{4}+241277 \lambda_{0}{ }^{5} \\
-30308 \lambda_{0}{ }^{6}+41488 \lambda_{0}{ }^{7}, \\
\gamma_{5}^{e}=-45616-256148 \lambda_{0}-80284 \lambda_{0}{ }^{2}+258176 \lambda_{0}{ }^{3}+338332 \lambda_{0}{ }^{4}+247844 \lambda_{0}{ }^{5} \\
\quad-21528 \lambda_{0}{ }^{6}+44496 \lambda_{0}{ }^{7}, \\
\gamma_{4}^{e}=93492-141885 \lambda_{0}-157892 \lambda_{0}{ }^{2}-62670 \lambda_{0}{ }^{3}+196340 \lambda_{0}{ }^{4}+561919 \lambda_{0}{ }^{5} \\
\quad-112884 \lambda_{0}{ }^{6}+91952 \lambda_{0}{ }^{7}, \\
\gamma_{3}^{e}=229024+57368 \lambda_{0}-632312 \lambda_{0}{ }^{2}-134528 \lambda_{0}{ }^{3}+353528 \lambda_{0}{ }^{4}-125816 \lambda_{0}{ }^{5} \\
+34128 \lambda_{0}{ }^{6}-3168 \lambda_{0}{ }^{7}, \\
\gamma_{2}^{e}=105828+271287 \lambda_{0}-114100 \lambda_{0}{ }^{2}-511698 \lambda_{0}{ }^{3}-136820 \lambda_{0}{ }^{4}+27623 \lambda_{0}{ }^{5} \\
\quad-50172 \lambda_{0}{ }^{6}+13360 \lambda_{0}{ }^{7}, \\
\gamma_{1}^{e}=-45616+384364 \lambda_{0}-221980 \lambda_{0}{ }^{2}-401920 \lambda_{0}{ }^{3}+356764 \lambda_{0}{ }^{4}-435292 \lambda_{0}{ }^{5} \\
+101736 \lambda_{0}{ }^{6}-47664 \lambda_{0}{ }^{7}, \\
\gamma_{0}^{e}=-13988+26457 \lambda_{0}+78804 \lambda_{0}{ }^{2}-101746 \lambda_{0}{ }^{3}-105876 \lambda_{0}{ }^{4}+48549 \lambda_{0}{ }^{5}
\end{gathered}
$$

B2.1

$$
\begin{aligned}
& \eta_{8}^{a}=\left(3888+20250 \lambda_{0}+35739 \lambda_{0}{ }^{2}+32424 \lambda_{0}{ }^{3}+26715 \lambda_{0}{ }^{4}+19834 \lambda_{0}{ }^{5}\right. \\
& +9764 \lambda_{0}{ }^{6}+7096 \lambda_{0}{ }^{7}+1536 \lambda_{0}{ }^{8}+1024 \lambda_{0}{ }^{9}, \\
& \eta_{7}^{a}=\left(7776+27864 \lambda_{0}+32760 \lambda_{0}{ }^{2}+10632 \lambda_{0}{ }^{3}+23256 \lambda_{0}{ }^{4}+16992 \lambda_{0}{ }^{5}\right. \\
& +5824 \lambda_{0}{ }^{6}+10816 \lambda_{0}{ }^{7}+1024 \lambda_{0}{ }^{8}+2048 \lambda_{0}{ }^{9}, \\
& \eta_{6}^{a}=\left(12312 \lambda_{0}+56700 \lambda_{0}{ }^{2}+53040 \lambda_{0}{ }^{3}+3516 \lambda_{0}{ }^{4}+21800 \lambda_{0}{ }^{5}\right. \\
& -4464 \lambda_{0}{ }^{6}+4704 \lambda_{0}{ }^{7}-512 \lambda_{0}{ }^{8}+1024 \lambda_{0}{ }^{9}, \\
& \eta_{5}^{a}=\left(7776+27864 \lambda_{0}+32760 \lambda_{0}{ }^{2}+10632 \lambda_{0}{ }^{3}+4824 \lambda_{0}{ }^{4}-19872 \lambda_{0}{ }^{5}\right. \\
& -5952 \lambda_{0}{ }^{6}-12736 \lambda_{0}{ }^{7}-1024 \lambda_{0}{ }^{8}-2048 \lambda_{0}{ }^{9}, \\
& \eta_{4}^{a}=-7776-15876 \lambda_{0}+41922 \lambda_{0}{ }^{2}+78096 \lambda_{0}{ }^{3}+27330 \lambda_{0}{ }^{4}+27484 \lambda_{0}{ }^{5} \\
& +18648 \lambda_{0}{ }^{6}-688 \lambda_{0}{ }^{7}+4096 \lambda_{0}^{8}, \\
& \eta_{3}^{a}=-7776-27864 \lambda_{0}-32760 \lambda_{0}{ }^{2}-10632 \lambda_{0}{ }^{3}-4824 \lambda_{0}{ }^{4}+19872 \lambda_{0}{ }^{5} \\
& +5952 \lambda_{0}{ }^{6}+12736 \lambda_{0}{ }^{7}+1024 \lambda_{0}{ }^{8}+2048 \lambda_{0}{ }^{9},
\end{aligned}
$$




$$
\begin{gathered}
\eta_{2}^{a}=12312 \lambda_{0}+56700 \lambda_{0}{ }^{2}+53040 \lambda_{0}{ }^{3}+3516 \lambda_{0}{ }^{4}+21800 \lambda_{0}{ }^{5} \\
-4464 \lambda_{0}{ }^{6}+4704 \lambda_{0}{ }^{7}-512 \lambda_{0}{ }^{8}+1024 \lambda_{0}{ }^{9} \\
\eta_{1}^{a}=-7776-27864 \lambda_{0}-32760 \lambda_{0}{ }^{2}-10632 \lambda_{0}{ }^{3}-23256 \lambda_{0}{ }^{4}-16992 \lambda_{0}{ }^{5} \\
-5824 \lambda_{0}{ }^{6}-10816 \lambda_{0}{ }^{7}-1024 \lambda_{0}{ }^{8}-2048 \lambda_{0}{ }^{9}, \\
\eta_{0}^{a}=3888+20250 \lambda_{0}+35739 \lambda_{0}{ }^{2}+32424 \lambda_{0}{ }^{3}+26715 \lambda_{0}{ }^{4}+19834 \lambda_{0}{ }^{5} \\
+9764 \lambda_{0}{ }^{6}+7096 \lambda_{0}{ }^{7}+1536 \lambda_{0}{ }^{8}+1024 \lambda_{0}{ }^{9} .
\end{gathered}
$$

\section{B2.2}

$$
\begin{aligned}
& \eta_{8}^{b}=-2160+11790 \lambda_{0}-21339 \lambda_{0}{ }^{2}+16563 \lambda_{0}{ }^{3}-9435 \lambda_{0}{ }^{4} \\
& +6210 \lambda_{0}{ }^{5}-1252 \lambda_{0}{ }^{6}+936 \lambda_{0}{ }^{7}, \\
& \eta_{7}^{b}=4320-14184 \lambda_{0}+21816 \lambda_{0}{ }^{2}-19332 \lambda_{0}{ }^{3}+7776 \lambda_{0}{ }^{4}-7728 \lambda_{0}{ }^{5} \\
& -32 \lambda_{0}{ }^{6}-960 \lambda_{0}{ }^{7} \\
& \eta_{6}^{b}=-504 \lambda_{0}-12924 \lambda_{0}{ }^{2}+23316 \lambda_{0}{ }^{3}+1140 \lambda_{0}{ }^{4}+7464 \lambda_{0}{ }^{5}+688 \lambda_{0}{ }^{6}+288 \lambda_{0}{ }^{7}, \\
& \eta_{5}^{b}=4320-14184 \lambda_{0}+7992 \lambda_{0}{ }^{2}+8316 \lambda_{0}{ }^{3}-1056 \lambda_{0}{ }^{4}+9936 \lambda_{0}{ }^{5} \\
& -1568 \lambda_{0}{ }^{6}+2112 \lambda_{0}{ }^{7}, \\
& \eta_{4}^{b}=4320+3060 \lambda_{0}-38466 \lambda_{0}{ }^{2}+31170 \lambda_{0}{ }^{3}-14178 \lambda_{0}{ }^{4}+5580 \lambda_{0}{ }^{5} \\
& -2264 \lambda_{0}{ }^{6}-1296 \lambda_{0}{ }^{7} \text {, } \\
& \eta_{3}^{b}=+14184 \lambda_{0}-7992 \lambda_{0}{ }^{2}-4320-8316 \lambda_{0}{ }^{3}+1056 \lambda_{0}{ }^{4}-9936 \lambda_{0}{ }^{5} \\
& +1568 \lambda_{0}{ }^{6}-2112 \lambda_{0}{ }^{7}, \\
& \eta_{2}^{b}=-504 \lambda_{0}-12924 \lambda_{0}{ }^{2}+23316 \lambda_{0}{ }^{3}+1140 \lambda_{0}{ }^{4}+7464 \lambda_{0}{ }^{5}+688 \lambda_{0}{ }^{6}+288 \lambda_{0}{ }^{7}, \\
& \eta_{1}^{b}=14184 \lambda_{0}-21816 \lambda_{0}{ }^{2}-4320+19332 \lambda_{0}{ }^{3}-7776 \lambda_{0}{ }^{4}+7728 \lambda_{0}{ }^{5} \\
& +32 \lambda_{0}{ }^{6}+960 \lambda_{0}{ }^{7}, \\
& \eta_{0}^{b}=-2160+11790 \lambda_{0}-21339 \lambda_{0}{ }^{2}+16563 \lambda_{0}{ }^{3}-9435 \lambda_{0}{ }^{4}+6210 \lambda_{0}{ }^{5} \\
& -1252 \lambda_{0}{ }^{6}+936 \lambda_{0}{ }^{7} .
\end{aligned}
$$

B2.3

$$
\begin{gathered}
\eta_{8}^{c}=-864+29541 \lambda_{0}-1831 \lambda_{0}{ }^{2}+32230 \lambda_{0}{ }^{3}+11103 \lambda_{0}{ }^{4}-40839 \lambda_{0}{ }^{5} \\
+27793 \lambda_{0}{ }^{6}-36652 \lambda_{0}{ }^{7}+18236 \lambda_{0}{ }^{8}-8704 \lambda_{0}{ }^{9}+3584 \lambda_{0}{ }^{10}, \\
\eta_{7}^{c}=5184-990 \lambda_{0}+54465 \lambda_{0}{ }^{2}+28110 \lambda_{0}{ }^{3}+74592 \lambda_{0}{ }^{4}+86883 \lambda_{0}{ }^{5} \\
-15230 \lambda_{0}{ }^{6}+55764 \lambda_{0}{ }^{7}-32200 \lambda_{0}{ }^{8}+10240 \lambda_{0}{ }^{9}-7168 \lambda_{0}{ }^{10}, \\
\eta_{6}^{c}=5184-40086 \lambda_{0}-61263 \lambda_{0}{ }^{2}-92125 \lambda_{0}{ }^{3}-123932 \lambda_{0}{ }^{4}-50616 \lambda_{0}{ }^{5} \\
-37600 \lambda_{0}{ }^{6}+6816 \lambda_{0}{ }^{7}+6624 \lambda_{0}{ }^{8}+2048 \lambda_{0}{ }^{9}+3584 \lambda_{0}{ }^{10}, \\
\eta_{5}^{c}=5184+25605 \lambda_{0}+3330 \lambda_{0}{ }^{2}+15437 \lambda_{0}{ }^{3}-22985 \lambda_{0}{ }^{4}-44499 \lambda_{0}{ }^{5} \\
-1770 \lambda_{0}{ }^{6}-43524 \lambda_{0}{ }^{7}+25400 \lambda_{0}{ }^{8}-13824 \lambda_{0}{ }^{9}+7168 \lambda_{0}{ }^{10},
\end{gathered}
$$




$$
\begin{aligned}
& \eta_{4}^{c}=10368+37089 \lambda_{0}+21138 \lambda_{0}{ }^{2}+84446 \lambda_{0}{ }^{3}+38999 \lambda_{0}{ }^{4}+10 \lambda_{0}{ }^{5} \\
& +66162 \lambda_{0}{ }^{6}-33864 \lambda_{0}{ }^{7}+23752 \lambda_{0}{ }^{8}-10752 \lambda_{0}{ }^{9}, \\
& \eta_{3}^{c}=12708 \lambda_{0}+58113 \lambda_{0}{ }^{2}+72304 \lambda_{0}{ }^{3}+127502 \lambda_{0}{ }^{4}+76145 \lambda_{0}{ }^{5} \\
& +21798 \lambda_{0}{ }^{6}+35452 \lambda_{0}{ }^{7}-25656 \lambda_{0}{ }^{8}-5184+10240 \lambda_{0}{ }^{9}-7168 \lambda_{0}{ }^{10}, \\
& \eta_{2}^{c}=-60648 \lambda_{0}-87115 \lambda_{0}{ }^{2}-129109 \lambda_{0}{ }^{3}-150338 \lambda_{0}{ }^{4}-46624 \lambda_{0}{ }^{5} \\
& -37688 \lambda_{0}{ }^{6}+9440 \lambda_{0}{ }^{7}+1728+8416 \lambda_{0}{ }^{8}+2048 \lambda_{0}{ }^{9}+3584 \lambda_{0}{ }^{10}, \\
& \eta_{1}^{c}=-13887 \lambda_{0}-56640 \lambda_{0}{ }^{2}-50959 \lambda_{0}{ }^{3}-87497 \lambda_{0}{ }^{4}-78937 \lambda_{0}{ }^{5} \\
& -7278 \lambda_{0}{ }^{6}-40364 \lambda_{0}{ }^{7}+25288 \lambda_{0}{ }^{8}-5184-6656 \lambda_{0}{ }^{9}+7168 \lambda_{0}{ }^{10}, \\
& \eta_{0}^{c}=-2592-1476 \lambda_{0}-31749 \lambda_{0}{ }^{2}-25334 \lambda_{0}{ }^{3}-37908 \lambda_{0}{ }^{4}-40795 \lambda_{0}{ }^{5} \\
& +5861 \lambda_{0}{ }^{6}-21772 \lambda_{0}{ }^{7}+14012 \lambda_{0}{ }^{8}-5120 \lambda_{0}{ }^{9}+3584 \lambda_{0}{ }^{10} .
\end{aligned}
$$

\section{B2.4}

$$
\begin{aligned}
& \eta_{8}^{d}=2160-40320 \lambda_{0}+215208 \lambda_{0}{ }^{2}-309507 \lambda_{0}{ }^{3}+187491 \lambda_{0}{ }^{4}-158277 \lambda_{0}{ }^{5} \\
& -160464 \lambda_{0}{ }^{6}+144658 \lambda_{0}{ }^{7}-139988 \lambda_{0}{ }^{8}+107216 \lambda_{0}{ }^{9}-26224 \lambda_{0}{ }^{10}+17280 \lambda_{0}{ }^{11}, \\
& \eta_{7}^{d}=-4320-10080 \lambda_{0}+342405 \lambda_{0}{ }^{2}-79980 \lambda_{0}{ }^{3}+127596 \lambda_{0}{ }^{4}+85401 \lambda_{0}{ }^{5} \\
& -188460 \lambda_{0}{ }^{6}+43302 \lambda_{0}{ }^{7}-106572 \lambda_{0}{ }^{8}-6688 \lambda_{0}{ }^{9}-17328 \lambda_{0}{ }^{10}-3008 \lambda_{0}{ }^{11}, \\
& \eta_{6}^{d}=-2160-2880 \lambda_{0}+116613 \lambda_{0}{ }^{2}+20787 \lambda_{0}{ }^{3}+170757 \lambda_{0}{ }^{4}-98394 \lambda_{0}{ }^{5} \\
& -167312 \lambda_{0}{ }^{6}+29792 \lambda_{0}{ }^{7}-145304 \lambda_{0}{ }^{8}+41552 \lambda_{0}{ }^{9}-27008 \lambda_{0}{ }^{10}+3776 \lambda_{0}{ }^{11}, \\
& \eta_{5}^{d}=2160+59040 \lambda_{0}-712305 \lambda_{0}{ }^{2}+1135929 \lambda_{0}{ }^{3}-585723 \lambda_{0}{ }^{4}+629508 \lambda_{0}{ }^{5} \\
& +442974 \lambda_{0}{ }^{6}-713132 \lambda_{0}{ }^{7}+528120 \lambda_{0}{ }^{8}-503024 \lambda_{0}{ }^{9}+110880 \lambda_{0}{ }^{10}-77504 \lambda_{0}{ }^{11} \text {, } \\
& \eta_{4}^{d}=-4320+175680 \lambda_{0}-1081809 \lambda_{0}{ }^{2}-26808 \lambda_{0}{ }^{3}-438462 \lambda_{0}{ }^{4}-371787 \lambda_{0}{ }^{5} \\
& +461862 \lambda_{0}{ }^{6}+206398 \lambda_{0}{ }^{7} 280380 \lambda_{0}{ }^{8}+258592 \lambda_{0}{ }^{9}+35664 \lambda_{0}{ }^{10}+48064 \lambda_{0}{ }^{11}, \\
& \eta_{3}^{d}=-84960 \lambda_{0}-352305 \lambda_{0}{ }^{2}-204618 \lambda_{0}{ }^{3}+17280-346362 \lambda_{0}{ }^{4}+208509 \lambda_{0}{ }^{5} \\
& +150720 \lambda_{0}{ }^{6}+38198 \lambda_{0}{ }^{7}+348372 \lambda_{0}{ }^{8}-27520 \lambda_{0}{ }^{9}+89424 \lambda_{0}{ }^{10}+1472 \lambda_{0}{ }^{11}, \\
& \eta_{2}^{d}=6480-190080 \lambda_{0}+1172655 \lambda_{0}{ }^{2}-714645 \lambda_{0}{ }^{3}+415425 \lambda_{0}{ }^{4}-193884 \lambda_{0}{ }^{5} \\
& -729924 \lambda_{0}{ }^{6}+673956 \lambda_{0}{ }^{7}-517136 \lambda_{0}{ }^{8}+461968 \lambda_{0}{ }^{9}-100000 \lambda_{0}{ }^{10}+80192 \lambda_{0}{ }^{11} \text {, } \\
& \eta_{1}^{d}=10800-154080 \lambda_{0}+391365 \lambda_{0}{ }^{2}+964113 \lambda_{0}{ }^{3}-75411 \lambda_{0}{ }^{4}+685074 \lambda_{0}{ }^{5} \\
& -178890 \lambda_{0}{ }^{6}-537080 \lambda_{0}{ }^{7}+63888 \lambda_{0}{ }^{8}-434352 \lambda_{0}{ }^{9}+34944 \lambda_{0}{ }^{10}-71488 \lambda_{0}{ }^{11} \text {, } \\
& \eta_{0}^{d}=6480-17280 \lambda_{0}-228771 \lambda_{0}{ }^{2}+84825 \lambda_{0}{ }^{3}-183519 \lambda_{0}{ }^{4}-64038 \lambda_{0}{ }^{5} \\
& +133078 \lambda_{0}{ }^{6}+2484 \lambda_{0}{ }^{7}+100336 \lambda_{0}{ }^{8}+63984 \lambda_{0}{ }^{9}+11008 \lambda_{0}{ }^{10}+18624 \lambda_{0}{ }^{11} .
\end{aligned}
$$

Acknowledgement The first and third authors are partially supported by NNSF of China grant No. 10671123. The second author is partially supported by a MCYT/FEDER grant No. MTM2005-06098-C02-01 and by a CICYT grant No. 2005SGR 00550. The third author 
is also partially supported by NCET of China grant No. 050391 and by SAB2006-0098 of Spain.

\section{REFERENCES}

[1] J. Chavarriga, H. Giacomini, J. Giné and J. Llibre, On the integrability of two-dimensional flows, J. Diff. Eqns. 157 (1999), 163-182.

[2] E. S. Cheb-Terrab And A. D. Roche, An Abel ODE class generalizing known integrable classes, Euro. J. Appl. Math. 14 (2003), 217-229.

[3] L. A. CheRKAs, The number of limit cycles of a centain second-order autonomous system, Differential Equations 12 (1976), 944-946.

[4] C. J. Christopher, Invariant algebraic curves and conditions for a center, Proc. Roy. Soc. Edinburgh Sect. A 124 (1994), 1209-1229.

[5] C. J. Christopher AND J. LliBRe, Integrability via invariant algebraic curves for planar polynomials differential systems, Ann. Diff. Eqns. 16 (2000), 5-19.

[6] C. B. Collins, Conditions for a center in a simple class of cubic systems, Differential Integral Equations 10 (1997), 333-356.

[7] R. Conti, Uniformly isochronous centers of polynomial systems in $\mathbb{R}^{2}$, in Differential Equations, Dynamical Systems, and Control Science, Lecture Notes in Pure and Appl. Math. Vol. 50, Dekker, New York, 1994, 21-31.

[8] J. Devlin, N. G. Lloyd And J. M. Pearson, Cubic systems and Abel equations, J. Diff. Eqns. 147 (1998), 435-454.

[9] M. V. Dolov, The canonical integral in the neighborhood of a focus, Differential Equations 12 (1976), 1946-1953.

[10] A. Gasull And J. Llibre, Limit cycles for a class of Abel equations, SIAM J. Math. Anal. 21 (1990), $1235-1244$

[11] A. Gasull, R. Prohens and J. Torregrosa, Limit cycles for rigid cubic systems, J. Math. Anal. Appl. 303 (2005), 391-404.

[12] H. Giacomini, J. Llibre And M. Viano, On the nonexistence, existence, and uniqueness of limit cycles, Nonlinearity 9 (1996), 501-516.

[13] J. Giné AND J. LLIBRE, Integrability and algebraic limit cycles for polynomial differential systems with homogeneous nonlinearities, J. Diff. Eqns. 208 (2005), 531-545.

[14] J. Giné And J. Llibre, A family of isochronous foci with Darboux first integral, Pacific J. Math. 218 (2005), 343-355.

[15] E. KAmKe, Differentialgleichungen "losungsmethoden und losungen", Col. Mathematik und ihre anwendungen, vol. 18, Akademische Verlagsgesellschaft Becker und Erler Kom-Ges., Leipzig, 1943.

[16] R. E. KooiJ And C. J. Christopher, Algebraic invariant curves and the integrability of polynomial systems, Appl. Math. Lett. 6 (1993), 51-53.

[17] C. Li, W. Li, J. Llibre, Z. Zhang, On the limit cycles of polynomial differential systems with homogeneous nonlinearities, Proc. Edinburgh Math. Soc. 43 (2000) 529-543.

[18] C. Li, W. Li, J. Llibre, Z. Zhang, New families of centers and limit cycles for polynomial differential systems with homogeneous nonlinearities, Ann. Differential Equations, 19 (2003), 302-317.

[19] A. Lins NeTo, Algebraic solutions of polynomial differential equations and foliations in dimension two, Lect. Notes Math. 1345 (1988), 192-232.

[20] J. Llibre And X. Zhang, Darboux integrability of real polynomial vector fields on regular algebraic hypersurfaces, Rendiconti Del Circolo Matematico Di Palermo, Serie II 51 (2002), 109-126.

[21] J. Llibre And X. Zhang, Invaraint algebraic surfaces of the Lorenz system, J. Math. Phys. 43 (2002), $1622-1645$. 
[22] N. G. Lloyd, C. J. Christopher, J. Devlin, J. M. Pearson and N. Yasmin, Quadratic-like cubic systems, Differential Equations Dynam. Systems 5 (1997), 329-345.

[23] G. Petit Bois, Tables of Indefinite Integrals, Dover Publications, Inc., New York, 1961.

[24] H. Poincaré, Sur líntégration des équations différentielles du premier ordre et du premier degré I and II, Rend. Circ. Mat. Palermo 5 (1891), 161-191; 11 (1897), 193-239.

[25] M. F. Singer, Liouvillian first integrals of differential equations, Trans. Amer. Math. Soc. 333 (1992), $673-688$.

[26] E. P. Volokitin, Center conditions for a simple class of quintic systems, Int. J. Math. Sci. 29 (2002), 625-632.

[27] Ye YanqIan, Theory of Limit Cycles, Translations of Math. Monographs, Vol. 66, Amer. Math. Soc., Providence, 1986.

[28] X. ZhAng, Invariant algebraic curves and rational first integrals of holomorphic foliations in $C P^{2}$, Sci. China Ser. A 46 (2003), 271-279.

[29] X. Zhang, Exponential factors and Darbouxian integrability for the Rössler system, Intern. J. Bifur. Chaos. 14 (2004), 4275-4283.

1 Department of Mathematics, Shanghai Jiaotong University, Shanghai, 200240, P. R. China

E-mail address: jinlong@sjtu.edu.cn, xzhang@sjtu.edu.cn

${ }^{2}$ Departament de Matemàtiques, Universitat Autònoma de Barcelona, 08193 Bellaterra, BarCELONA, Spain

E-mail address: jllibre@mat.uab.cat 